\title{
Critical Neumann Problem with Competing Hardy Potentials
}

\author{
Daomin CAO and Jan CHABROWSKI
}

\author{
Institute of Applied Mathematics \\ Academy of Mathematics and Systems Science \\ Chinese Academy of Sciences \\ Beijing 100080 - PR China \\ dmcao@amt.ac.cn
}

\author{
Department of Mathematics \\ University of Queensland \\ St. Lucia 4072, QId — Australia \\ jhc@maths . uq. edu . au
}

Received: September 9, 2006

Accepted: March 1, 2007

\begin{abstract}
In this paper we investigate the solvability of the nonlinear Neumann problem (1) involving a critical Sobolev nonlinearity and two competing Hardy potentials in a bounded domain. We examine the common effect of the shape of the graph of the weight function, the mean curvature of the boundary and Hardy potentials on the existence of solutions of problem (1). We are mainly interested in the existence of positive solutions. We also obtain the existence of sign-changing solutions.
\end{abstract}

Key words: critical Sobolev exponent, Hardy potential, concentration-compactness principle, Neumann problem.

2000 Mathematics Subject Classification: 35B33, 35J65, 35Q55.

\section{Introduction}

In this paper we investigate the existence of solutions of the problem

$$
\begin{cases}-\Delta u+\lambda \frac{u}{|x-a|^{2}}-\gamma \frac{u}{|x|^{2}} & =Q(x)|u|^{2^{*}-2} u \text { in } \Omega, \\ \frac{\partial u}{\partial \nu} & =0 \text { on } \partial \Omega, \quad u>0 \text { on } \Omega,\end{cases}
$$

where $\Omega \subset \mathbb{R}^{N}$ is a bounded domain with a smooth boundary, $\lambda>0$ and $\gamma>0$ are parameters, $N \geq 3,2^{*}=\frac{2 N}{N-2}$ is a critical Sobolev exponent and $Q$ is a positive 
continuous function on $\bar{\Omega}$. $\nu$ denotes an outward normal vector to $\partial \Omega$. We assume that 0 and $a$ belong to $\partial \Omega$ and $a \neq 0$.

A function $u \in H^{1}(\Omega)$ is said to be a solution of (1) if $u$ satisfies

$$
\int_{\Omega}\left(\nabla u \nabla v+\lambda \frac{u v}{|x-a|^{2}}-\gamma \frac{u v}{|x|^{2}}\right) d x=\int_{\Omega} Q(x)|u|^{2^{*}-2} u v d x
$$

for every $v \in H^{1}(\Omega)$. Solutions to (1) are obtained as critical points of the variational functional

$$
J_{\lambda, \gamma}(u)=\frac{1}{2} \int_{\Omega}\left(|\nabla u|^{2}+\frac{\lambda u^{2}}{|x-a|^{2}}-\frac{\gamma u^{2}}{|x|^{2}}\right) d x-\frac{1}{2^{*}} \int_{\Omega} Q(x)|u|^{2^{*}} d x .
$$

Since the terms $\int_{\Omega} \frac{u^{2}}{|x-a|^{2}} d x$ and $\int_{\Omega} \frac{u^{2}}{|x|^{2}} d x$ satisfy an Hardy type inequality (see section 1) it is easy to see that $J_{\lambda, \gamma}$ is a $C^{1}$-functional on $H^{1}(\Omega)$. We are primarily interested in the existence of positive solutions. We also establish in one case the existence of a sign - changing solution. If $\lambda=0$ and $\gamma>0$ then problem (1) does not have a positive solution. Indeed, if $u>0$ is a solution of (1), then testing (2) with $v=1$ we obtain

$$
-\int_{\Omega} \frac{\gamma u}{|x|^{2}} d x=\int_{\Omega} Q(x)|u|^{2^{*}-2} u d x
$$

which is impossible. On the other hand we will show that if $\gamma=0$ and $\lambda>0$, then problem (1) has a positive solution which in fact is a least energy solution. This will be proved under some assumptions on the graph of the coefficient $Q$. In this paper we discuss the existence of solutions in the presence of two competing Hardy potentials. The Dirichlet problem involving the Hardy potentials in recent years has attracted a considerable interest $[6,7,12,17,18,25,30]$. In these papers the existence of positive and possibly multiple solutions have been established. A large part of these results have been extended to a nonlinear critical problem involving the $p$-Laplacian [20,23]. However much less is known for the Neumann problem involving the Hardy potential. Some existence results can be found in the papers $[10,19,23]$. The paper [10] considers the case where the singularity of the Hardy potential is in the interior of $\Omega$. In papers $[19,23]$ the singular points of the Hardy potential belong to $\partial \Omega$. As in the case of the Dirichlet problem, the Neumann problem (1) is more interesting when $0, a$ belong to $\partial \Omega[16]$. The paper [23] deals mainly with the critical problem with the mixed boundary conditions. The authors of [19] prove the existence of positive solutions for the nonlinear Neumann problem with the Hardy-Sobolev potential.

Let $\phi: X \rightarrow \mathbb{R}$ be a $C^{1}$ functional on a Banach space $X$. We recall that a sequence $\left\{x_{n}\right\} \subset X$ is a Palais-Smale sequence for $\phi$ at level $c$ (a (PS) $)_{c}$ sequence for short) if $\phi\left(x_{n}\right) \rightarrow c$ and $\phi^{\prime}\left(x_{n}\right) \rightarrow 0$ in $X^{*}$. Finally, we say that the functional $\phi$ satisfies the Palais-Smale condition at level $c\left((\mathrm{PS})_{c}\right.$ condition for short) if each $(\mathrm{PS})_{c}$ sequence is relatively compact in $X$.

Throughout this paper we denote a strong convergence by " $\rightarrow$ " and a weak convergence by "—". The norms in the Lebesgue spaces $L^{p}\left(\mathbb{R}^{N}\right)$ are denoted by $\|\cdot\|_{p}$. 
The paper is organized as follows. In section 1 we find the energy level for the functional $J_{\lambda, \gamma}$ below which the Palais-Smale condition holds. Our approach is based on the P. L. Lions concentration-compactness principle [26]. Lemma 1.1, which extends the Hardy inequality to functions in $H^{1}(\Omega)$, is crucial in our approach. It allows us to show that the quadratic part of the functional $J_{\lambda, \gamma}$ is positive definite, despite the fact that the coefficient $\left(\frac{\lambda}{|x-a|^{2}}-\frac{\gamma}{|x|^{2}}\right)$ is neither bounded above nor below. Section 2 contains the main results on the existence of positive solutions. These solutions are obtained by the mountain-pass principle. Section 3 is devoted to the regularity of solutions of problem (1). We point out here that solutions have only a singularity at 0 . The presence of the Hardy potential with a parameter $\lambda>0$ does not create a singularity at $a$. In section 4 we establish the existence of sign-changing solutions of (1). The main results of this paper are Theorems 2.1, 2.2, 2.3 giving the existence of positive solutions of problem (1) and Theorem 4.4 establishing the existence of a sign-changing solution of problem (1). Unlike in the case of positive solutions, we have only established the existence of sign-changing solutions in the case (I) (see section 3 ). The difficulty arises in estimating a min-max level in Lemma 4.3, which seems to be possible only in this case.

\section{Preliminaries}

It is well known that if $0 \in \Omega$ then for every $u \in H_{\circ}^{1}(\Omega)$ the following Hardy inequality holds

$$
\int_{\Omega} \frac{u^{2}}{|x|^{2}} d x \leq \frac{1}{\bar{\mu}} \int_{\Omega}|\nabla u|^{2} d x
$$

where $\bar{\mu}=\frac{(N-2)^{2}}{4}$ is an optimal constant. In this paper we need an extension of (3) to functions in $H^{1}(\Omega)$ assuming that $0 \in \partial \Omega$. For the case $0 \in \Omega$ we refer to the paper [10].

Lemma 1.1. Let $0 \in \partial \Omega$. Then for every $\epsilon>0$ there exists a constant $C(\epsilon)>0$ such that

$$
\int_{\Omega} \frac{u^{2}}{|x|^{2}} d x \leq\left(\frac{1}{\bar{\mu}}+\epsilon\right) \int_{\Omega}|\nabla u|^{2} d x+C(\epsilon) \int_{\Omega} u^{2} d x
$$

for every $u \in H^{1}(\Omega)$.

Proof. We partly follow the argument from [31, Lemma 2.1]. Through translations and rotations of the coordinate system we may assume that the inner normal to $\partial \Omega$ at 0 is pointing in the direction of the positive $x_{N}$-axis. Then there exists a smooth function $\psi\left(x^{\prime}\right), x^{\prime}=\left(x_{1}, \ldots, x_{N-1}\right)$, defined for $\left|x^{\prime}\right|$ small with $\psi$ and $\nabla \psi$, vanishing at $0^{\prime}$ such that $A=\Omega \cap B\left(0, \delta_{\circ}\right)=\left\{\left(x^{\prime}, x_{N}\right) \in B\left(0, \delta_{\circ}\right), x_{N}>\psi\left(x^{\prime}\right)\right\}$ and $\partial \Omega \cap B\left(0, \delta_{\circ}\right)=$ $\left\{\left(x^{\prime}, x_{N}\right) \in B\left(0, \delta_{\circ}\right), x_{N}=\psi\left(x^{\prime}\right)\right\}$. First we consider the case $\psi \equiv 0$. For every $u \in H^{1}\left(B\left(0, \delta_{\circ}\right)\right)$ with supp $u \subset B\left(0, \delta_{\circ}\right)$ we have

$$
\int_{A}|\nabla u|^{2} d x \geq \bar{\mu} \int_{A} \frac{u^{2}}{|x|^{2}} d x .
$$


Since the values of $u$ for $x_{N}<0$ are irrelevant, we may assume that $u$ is an even function in $x_{N}$. Thus

$$
\int_{A}|\nabla u|^{2} d x=\frac{1}{2} \int_{B\left(0, \delta_{\circ}\right)}|\nabla u|^{2} d x \geq \frac{\bar{\mu}}{2} \int_{B\left(0, \delta_{\circ}\right)} \frac{u^{2}}{|x|^{2}} d x=\bar{\mu} \int_{A} \frac{u^{2}}{|x|^{2}} d x .
$$

If $\psi \not \equiv 0$, we use the transformation (see [27]) which straightens the boundary $\partial \Omega$ around 0 : for $|y|$ sufficiently small we define a mapping $x=\Phi(y)=\left(\Phi_{1}(y), \ldots, \Phi_{N}(y)\right)$ by

$$
\Phi_{j}(y)=\left\{\begin{array}{lll}
y_{j}-y_{N} \frac{\partial \psi}{\partial x_{j}} & \text { for } \quad j=1, \ldots, N-1, \\
y_{N}+\psi\left(y^{\prime}\right) & \text { for } \quad j=N .
\end{array}\right.
$$

Since $\nabla \psi(0)=0$, the differential mapping $D \Phi$ of $\Phi$ satisfies that $D \Phi(0)$ is the identity mapping. Hence $\Phi$ has an inverse mapping $y=\Phi^{-1}(x)$ for $|x| \leq \delta, \delta \leq \delta_{\circ}$. We use notation $\Psi(x)=\Phi^{-1}(x)$. It follows from [27, Lemma A.1] that

$$
\operatorname{det} D \Phi(y)=1-\alpha y_{N}+O\left(|y|^{2}\right),
$$

where $\alpha=\Delta \psi(0)$ and moreover we have from formulae [27, (A.6)]

$$
\begin{aligned}
\frac{\partial \Psi_{i}}{\partial x_{j}}(\Phi(y)) & =\delta_{i j}+\frac{\partial^{2} \psi\left(y^{\prime}\right)}{\partial x_{i} \partial x_{j}} y_{N}+O\left(|y|^{2}\right), \quad i, j=1, \ldots, N-1, \\
\frac{\partial \Psi_{i}}{\partial x_{N}}(\Phi(y)) & =\frac{\partial \psi\left(y^{\prime}\right)}{\partial x_{i}}+O\left(|y|^{2}\right), \quad i=1, \ldots, N-1, \\
\frac{\partial \Psi_{N}}{\partial x_{j}}(\Phi(y)) & =-\frac{\partial \psi\left(y^{\prime}\right)}{\partial x_{j}}+O\left(|y|^{2}\right), \quad j=1, \ldots, N-1, \\
\frac{\partial \Psi_{N}}{\partial x_{N}}(\Phi(y)) & =1+O\left(|y|^{2}\right) .
\end{aligned}
$$

Using the mean value theorem we deduce from this that

$$
|y|^{2}=|\Psi(x)|^{2} \leq|x|^{2}\left(1+\epsilon_{1}(\delta)\right),
$$

where $\epsilon_{1}(\delta) \rightarrow 0$ as $\delta \rightarrow 0$. By the first part of the proof the function $v(y)=u(\Phi(y))$, $x \in A$, satisfies the inequality

$$
\int_{A} \frac{v(y)^{2}}{|y|^{2}} d y \leq \frac{1}{\bar{\mu}} \int_{A}|\nabla v(y)|^{2} d y .
$$

To change variables $y=\Psi(x)$ in the above integrals we first observe that

$$
|D \Psi(x)|^{2} \leq 1+\epsilon_{2}(\delta),
$$

where $\epsilon_{2}(\delta) \rightarrow 0$ as $\delta \rightarrow 0$. Thus

$$
\int_{\Omega \cap B(0, \delta)} \frac{u(x)^{2}}{|x|^{2}} d x \leq \frac{1}{\bar{\mu}}\left(1+\epsilon_{1}(\delta)\right)\left(1+\epsilon_{2}(\delta)\right) M(\delta) \int_{\Omega \cap B(0, \delta)}|\nabla u(x)|^{2} d x,
$$


where

$$
M(\delta)=\frac{\max _{\Omega \cap B(0, \delta)}|\operatorname{det} D \Psi(x)|}{\min _{\Omega \cap B(0, \delta)}|\operatorname{det} D \Psi(x)|} .
$$

By (5) we see that $M(\delta) \leq 1+\epsilon_{3}(\delta)$, with $\epsilon_{3}(\delta) \rightarrow 0$ as $\delta \rightarrow 0$. Given $\epsilon>0$ we can choose $\delta=\delta(\epsilon)>0$ such that

$$
\frac{1}{\bar{\mu}}\left(1+\epsilon_{1}(\delta)\right)\left(1+\epsilon_{2}(\delta)\right)\left(1+\epsilon_{3}(\delta)\right) \leq \frac{1}{\bar{\mu}}+\epsilon .
$$

Finally, let $\phi$ be a $C_{\circ}^{1}(B(0, \delta))$ function such that $\phi(x)=1$ for $x$ near 0 and $0 \leq \phi(x) \leq 1$ on $B(0, \delta)$. Then

$$
\begin{aligned}
\int_{\Omega} \frac{u^{2}}{|x|^{2}} d x & =\int_{\Omega} \frac{(u \phi)^{2}}{|x|^{2}} d x+\int_{\Omega} \frac{\left(1-\phi^{2}\right) u^{2}}{|x|^{2}} d x \\
& \leq\left(\frac{1}{\bar{\mu}}+\frac{\epsilon}{2}\right) \int_{\Omega}|\nabla(u \phi)|^{2} d x+\int_{\Omega} \frac{\left(1-\phi^{2}\right)}{|x|^{2}} u^{2} d x .
\end{aligned}
$$

Estimate (4) follows from (6) and the Young inequality.

Remark 1.2. Estimate (6) implies the following refinement of (4): for every $\epsilon>0$ there exist constants $\rho(\epsilon)>0$ and $C(\epsilon)>0$ such that

$$
\int_{\Omega} \frac{u^{2}}{|x|^{2}} d x \leq\left(\frac{1}{\bar{\mu}}+\epsilon\right) \int_{\Omega \cap B(0, \rho(\epsilon))}|\nabla u|^{2} d x+C(\epsilon) \int_{\Omega} u^{2} d x
$$

for every $u \in H^{1}(\Omega)$.

Lemma 1.3. Let $\left\{u_{m}\right\} \subset H^{1}(\Omega)$ be a $(\mathrm{PS})_{c}$ sequence for the functional $J_{\lambda, \gamma}$. If $\gamma<\bar{\mu}$, then $\left\{u_{m}\right\}$ is bounded in $H^{1}(\Omega)$.

Proof. We have

$$
J_{\lambda, \gamma}\left(u_{m}\right)-\frac{1}{2}\left\langle J_{\lambda, \gamma}^{\prime}\left(u_{m}\right), u_{m}\right\rangle=\frac{1}{N} \int_{\Omega} Q(x)\left|u_{m}\right|^{2^{*}} d x .
$$

Hence

$$
\int_{\Omega}\left|u_{m}\right|^{2^{*}} d x \leq \frac{1}{Q_{*}} \int_{\Omega} Q(x)\left|u_{m}\right|^{2^{*}} d x \leq \frac{N c}{Q_{*}}+1+o\left(\left\|u_{m}\right\|_{H^{1}}\right)
$$

for $m \geq m_{\circ}$, where $Q_{*}=\min _{x \in \bar{\Omega}} Q(x)$. From this, using the Young inequality we deduce that

$$
\int_{\Omega} u_{m}^{2} d x \leq \frac{2}{2^{*}} \int_{\Omega}\left|u_{m}\right|^{2^{*}} d x+\frac{2^{*}-2}{2^{*}}|\Omega| \leq C_{1}+o\left(\left\|u_{m}\right\|_{H^{1}}\right)
$$

for some constant $C_{1}>0$ independent of $m$. Since $J_{\lambda, \gamma}\left(u_{m}\right) \rightarrow c$ we see that

$$
\int_{\Omega}\left(\left|\nabla u_{m}\right|^{2}-\gamma \frac{u_{m}^{2}}{|x|^{2}}\right) d x \leq \int_{\Omega}\left(\left|\nabla u_{m}\right|^{2}+\frac{\lambda u_{m}^{2}}{|x-a|^{2}}-\frac{\gamma u_{m}^{2}}{|x|^{2}}\right) d x \leq C_{2}+o\left(\left\|u_{m}\right\|_{H^{1}}\right),
$$


where $C_{2}>0$ is a constant independent of $m$. We now choose $\epsilon>0$ so that $\left(\frac{1}{\bar{\mu}}+\epsilon\right) \gamma$ $<1$. By Lemma 1.1 there exists a constant $C(\epsilon)>0$ such that

$$
\left(1-\left(\frac{1}{\bar{\mu}}+\epsilon\right) \gamma\right) \int_{\Omega}\left|\nabla u_{m}\right|^{2} d x \leq C_{2}+C(\epsilon) \int_{\Omega} u_{m}^{2} d x+o\left(\left\|u_{m}\right\|_{H^{1}}\right) .
$$

This combined together with (7) shows that $\left\{u_{m}\right\}$ is bounded in $H^{1}(\Omega)$.

To proceed further, we put $Q_{m}=\max _{x \in \partial \Omega} Q(x)$ and $Q_{M}=\max _{x \in \bar{\Omega}} Q(x)$. To find the level $c$ below which the (PS) condition holds, we use the following constant

$$
S_{-\gamma}=\inf _{u \in \mathcal{D}^{1,2}\left(\mathbb{R}^{N}\right)-\{0\}} \frac{\int_{\mathbb{R}^{N}}\left(|\nabla u|^{2}-\frac{\gamma u^{2}}{|x|^{2}}\right) d x}{\left(\int_{\mathbb{R}^{N}}|u|^{2^{*}} d x\right)^{\frac{2}{2^{*}}}}
$$

for $0<\gamma<\bar{\mu}$, where $\mathcal{D}^{1,2}\left(\mathbb{R}^{N}\right)$ is a Sobolev space defined as the closure of $C_{\circ}^{\infty}\left(\mathbb{R}^{N}\right)$ with respect to the norm $\|u\|_{\mathcal{D}^{1,2}}^{2}=\int_{\mathbb{R}^{N}}|\nabla u|^{2} d x$. It is known [30] that $S_{-\gamma}$ is attained by the function (see also $[9,24]$ )

$$
U_{\gamma}=\frac{(4 N(\bar{\mu}-\gamma)(N-2))^{\frac{N-2}{4}}}{\left(|x|^{\frac{\gamma^{\prime}}{\sqrt{\bar{\mu}}}}+|x|^{\frac{\bar{\gamma}}{\sqrt{\bar{\mu}}}}\right)^{\sqrt{\bar{\mu}}}},
$$

where $\bar{\gamma}=\sqrt{\bar{\mu}}+\sqrt{\bar{\mu}-\gamma}$ and $\gamma^{\prime}=\sqrt{\bar{\mu}}-\sqrt{\bar{\mu}-\gamma}$. For $\epsilon>0$ we put

$$
V_{\gamma, \epsilon}(x)=\frac{(4 N(\bar{\mu}-\gamma)(N-2))^{\frac{N-2}{4}} \epsilon^{\frac{N-2}{2}}}{\left(\epsilon^{2}|x|^{\frac{\gamma^{\prime}}{\sqrt{\bar{\mu}}}}+|x|^{\frac{\gamma}{\sqrt{\bar{\mu}}}}\right)^{\sqrt{\bar{\mu}}}} .
$$

The function $V_{\gamma, \epsilon}$ is a solution of the problem

$$
\begin{cases}-\Delta u-\gamma \frac{u}{|x|^{2}}=|u|^{2^{*}-2} u & \text { in } \mathbb{R}^{N}-\{0\}, \\ u(x) \rightarrow 0 & \text { as }|x| \rightarrow \infty\end{cases}
$$

We also define $S_{\lambda}$, with $\lambda>0$, by

$$
S_{\lambda}=\inf _{u \in \mathcal{D}^{1,2}\left(\mathbb{R}^{N}\right)-\{0\}} \frac{\int_{\mathbb{R}^{N}}\left(|\nabla u|^{2}+\lambda \frac{u^{2}}{|x|^{2}}\right) d x}{\left(\int_{\mathbb{R}^{N}}|u|^{2^{*}} d x\right)^{\frac{2}{2^{2}}}} .
$$

It is obvious that $S_{\lambda} \geq S$, where $S$ is a usual Sobolev constant defined by

$$
S=\inf _{u \in \mathcal{D}^{1,2}\left(\mathbb{R}^{N}\right)-\{0\}} \frac{\int_{\mathbb{R}^{N}}|\nabla u|^{2} d x}{\left(\int_{\mathbb{R}^{N}}|u|^{2^{*}} d x\right)^{\frac{2}{2^{*}}}} .
$$


In paper [30] it was shown that

$$
S_{-\gamma}=S\left(1-\frac{4 \gamma}{(N-2)^{2}}\right)^{\frac{N-1}{N}}
$$

Proposition 1.4. Suppose that $\gamma<\bar{\mu}$. If $\left\{u_{m}\right\}$ is a $(\mathrm{PS})_{c}$ sequence for $J_{\lambda, \gamma}$ with

$$
c<\frac{1}{N} \min \left(\frac{S_{-\gamma}^{\frac{N}{2}}}{2 Q(0)^{\frac{N-2}{2}}}, \frac{S^{\frac{N}{2}}}{2 Q_{m^{\frac{N-2}{2}}}}, \frac{S^{\frac{N}{2}}}{Q_{M}^{\frac{N-2}{2}}}\right),
$$

then $\left\{u_{m}\right\}$ is relatively compact in $H^{1}(\Omega)$.

Proof. Since $\left\{u_{m}\right\}$ is bounded in $H^{1}(\Omega)$ we can assume that

$$
\begin{gathered}
u_{m} \rightarrow u \text { in } H^{1}(\Omega), \quad u_{m} \rightarrow u \text { in } L^{2^{*}}(\Omega), \\
u_{m} \rightarrow u \text { in } L^{2}\left(\Omega, \frac{d x}{|x-a|^{2}}\right), \quad \text { and } \quad u_{m} \rightarrow u \text { in } L^{2}\left(\Omega, \frac{d x}{|x|^{2}}\right) .
\end{gathered}
$$

By the concentration-compactness principle [26] there exist at most countable set $\mathcal{J}$, a set of distinct points $\left\{x_{j}\right\}, j \in \mathcal{J} \subset \bar{\Omega}-(\{a\},\{0\})$ and numbers $\mu_{j}, \nu_{j}, j \in \mathcal{J}, \mu_{a}$, $\mu_{0}, \nu_{a}, \nu_{0}, \gamma_{a}, \lambda_{0}$ such that

$$
\begin{gathered}
\left|\nabla u_{m}\right|^{2} d x \rightarrow d \mu \geq|\nabla u|^{2} d x+\sum_{j \in \mathcal{J}} \mu_{j} \delta_{x_{j}}+\mu_{a} \delta_{a}+\mu_{0} \delta_{0}, \\
\left|u_{m}\right|^{2^{*}} d x \rightarrow d \nu=|u|^{2^{*}} d x+\sum_{j \in \mathcal{J}} \nu_{j} \delta_{x_{j}}+\nu_{a} \delta_{a}+\nu_{0} \delta_{0},
\end{gathered}
$$

and

$$
\begin{aligned}
\frac{u_{m}^{2}}{|x-a|^{2}} d x \rightarrow d \tilde{\gamma} & =\frac{u^{2}}{|x-a|^{2}} d x+\gamma_{a} \delta_{a}, \\
\frac{u_{m}^{2}}{|x|^{2}} d x \rightarrow d \tilde{\lambda} & =\frac{u^{2}}{|x|^{2}} d x+\lambda_{0} \delta_{0} .
\end{aligned}
$$

From the Sobolev inequality we have

$$
\begin{gathered}
S \nu_{j}^{\frac{2}{2^{*}}} \leq \mu_{j} \quad \text { if } x_{j} \in \Omega \\
\frac{S}{2^{\frac{2}{N}}} \nu_{j}^{\frac{2}{2^{*}}} \leq \mu_{j} \quad \text { if } x_{j} \in \partial \Omega \\
\frac{S_{\lambda}}{2^{\frac{2}{N}}} \nu_{a}^{\frac{2}{2^{*}}} \leq \mu_{a}+\lambda \gamma_{a} \\
\frac{S_{-\gamma}}{2^{\frac{2}{N}}} \nu_{0}^{\frac{2}{2^{*}}} \leq \mu_{0}-\gamma \lambda_{0} .
\end{gathered}
$$


As in [6] we show that $\mathcal{J}$ is a finite set and

$$
\mu_{j} \leq Q\left(x_{j}\right) \nu_{j}, \quad j \in \mathcal{J} .
$$

If $\nu_{j}>0$ and $x_{j} \in \Omega$, then by (9) and (13) we obtain

$$
\frac{S^{\frac{N}{2}}}{Q\left(x_{j}\right)^{\frac{N}{2}}} \leq \nu_{j} .
$$

If $\nu_{j}>0$ and $x_{j} \in \partial \Omega$, then (10) and (13) yield

$$
\frac{S^{\frac{N}{2}}}{2 Q\left(x_{j}\right)^{\frac{N}{2}}} \leq \nu_{j} .
$$

On the other hand we have

$$
\mu_{a} \leq Q(a) \nu_{a}-\lambda \gamma_{a} \quad \text { and } \quad \mu_{0} \leq Q(0) \nu_{0}+\gamma \lambda_{0} .
$$

Hence, if $\nu_{a}>0$ then using (11) we derive

$$
\frac{S_{\lambda}^{\frac{N}{2}}}{2 Q(a)^{\frac{N}{2}}} \leq \nu_{a} .
$$

Finally, if $\nu_{0}>0$, then (12) yields

$$
\frac{S_{-\gamma}^{\frac{N}{2}}}{2 Q(0)^{\frac{N}{2}}} \leq \nu_{0}
$$

We now observe that

$$
\begin{aligned}
c & =J_{\lambda, \gamma}\left(u_{m}\right)-\frac{1}{2}\left\langle J_{\lambda, \gamma}^{\prime}\left(u_{m}\right), u_{m}\right\rangle+o(1)=\frac{1}{N} \int_{\Omega} Q(x)\left|u_{m}\right|^{2^{*}} d x+o(1) \\
& =\frac{1}{N}\left(\int_{\Omega} Q(x)|u|^{2^{*}} d x+\sum_{j \in \mathcal{J}} Q\left(x_{j}\right) \nu_{j}+Q(0) \nu_{0}+Q(a) \nu_{a}\right) .
\end{aligned}
$$

If there exists $j \in \mathcal{J}$ such that $\nu_{j}>0$ (with $x_{j} \in \Omega$ or $x_{j} \in \partial \Omega$ ) or $\nu_{a}>0$ or $\nu_{0}>0$, then by (14), (15), (17), and (18) we get

$$
c \geq \frac{1}{N} \min \left(\frac{S_{\lambda}^{\frac{N}{2}}}{2 Q(a)^{\frac{N-2}{2}}}, \frac{S_{-\gamma}^{\frac{N}{2}}}{2 Q(0)^{\frac{N-2}{2}}}, \frac{S^{\frac{N}{2}}}{2 Q_{m^{2}}^{\frac{N-2}{2}}}, \frac{S^{\frac{N}{2}}}{Q_{M}^{\frac{N-2}{2}}}\right) .
$$

Since $S_{\lambda} \geq S$ and $Q(a) \leq Q_{m}$ we deduce from the above inequality that

$$
c \geq \frac{1}{N} \min \left(\frac{S_{-\gamma}^{\frac{N}{2}}}{2 Q(0)^{\frac{N-2}{2}}}, \frac{S^{\frac{N}{2}}}{2 Q_{m^{2}}^{\frac{N-2}{2}}}, \frac{S^{\frac{N}{2}}}{Q_{M}^{\frac{N-2}{2}}}\right) .
$$


This is impossible. Therefore $\nu_{j}=0, j \in \mathcal{J}, \nu_{a}=\nu_{0}=0$. Then (13) yields $\mu_{j}=0$, $j \in \mathcal{J}$. By the first inequality in (16) we get $\gamma_{a}=0$. The second inequality in (16) combined with Lemma 1.1 and the assumption $\gamma<\bar{\mu}$ yields $\lambda_{0}=0$. This shows that $\left\{u_{m}\right\}$ is relatively compact in $H^{1}(\Omega)$.

We now establish the mountain-pass geometry for the functional $J_{\lambda, \gamma}$.

Proposition 1.5. For every $\lambda>0$ there exists $\gamma_{0}=\gamma_{0}(\lambda)>0$ such that for $\gamma \leq \gamma_{0}$ there exist constants $\beta>0$ and $\rho>0$ such that

$$
J_{\lambda, \gamma}(u) \geq \beta \text { for }\|u\|_{H^{1}(\Omega)}=\rho .
$$

Proof. Let $d=\operatorname{diam} \Omega$. We then have

$$
J_{\lambda, \gamma}(u) \geq \frac{1}{2} \int_{\Omega}\left(|\nabla u|^{2}+\frac{\lambda}{d^{2}} u^{2}\right) d x-\frac{\gamma}{2} \int_{\Omega} \frac{u^{2}}{|x|^{2}} d x-\frac{1}{2^{*}} \int_{\Omega} Q(x)|u|^{2^{*}} d x .
$$

First we make the restriction $\frac{\gamma}{\bar{\mu}}<1$. We choose $\epsilon>0$ so that $\gamma\left(\frac{1}{\bar{\mu}}+\epsilon\right)<1$. By Lemma 1.1 and the Sobolev inequality there exist constants $C(\epsilon)>0$ and $C_{1}=$ $C\left(|\Omega|, Q_{M}, N\right)>0$ such that

$$
\begin{aligned}
J_{\lambda, \gamma}(u) \geq \frac{1}{2} \int_{\Omega}\left(|\nabla u|^{2}+\frac{\lambda u^{2}}{d^{2}}\right) d x-\frac{\gamma}{2}\left(\frac{1}{\bar{\mu}}+\epsilon\right) \int_{\Omega}|\nabla u|^{2} d x-\frac{C(\epsilon) \gamma}{2} \int_{\Omega} u^{2} d x & \\
& -C_{1}\left(\int_{\Omega}\left(|\nabla u|^{2}+u^{2}\right) d x\right)^{\frac{2^{*}}{2}} .
\end{aligned}
$$

We now choose $\gamma_{\circ}$, smaller if necessary, to ensure that $C(\epsilon) \gamma<\frac{\lambda}{d^{2}}$ for $\gamma \leq \gamma_{\circ}$. Hence

$$
J_{\lambda, \gamma}(u) \geq c_{1} \int_{\Omega}\left(|\nabla u|^{2}+u^{2}\right) d x-C_{1}\left(\int_{\Omega}\left(|\nabla u|^{2}+u^{2}\right) d x\right)^{\frac{2^{*}}{2}},
$$

where $c_{1}=\min \left(\frac{1}{2}-\frac{\gamma}{2}\left(\frac{1}{\bar{\mu}}+\epsilon\right), \frac{\lambda}{2 d^{2}}-\frac{C(\epsilon) \gamma}{2}\right)$. Let $\rho^{2}=\|u\|_{H^{1}(\Omega)}^{2}$. Taking $\rho>0$ small enough, the result follows.

\section{Existence of positive solutions}

We will obtain solutions of problem (1) using the mountain-pass principle. We put

$$
\tilde{c}=\inf _{g \in \Gamma} \max _{t \in[0,1]} J_{\lambda, \gamma}(g(t)),
$$

where $\Gamma=\left\{g \in C\left([0,1], H^{1}(\Omega)\right) ; g(0)=0, g(1)=v\right\}$, with $v \in H^{1}(\Omega)$ chosen so that $\|v\|_{H^{1}(\Omega)}>\rho$ and $J_{\lambda, \gamma}(v)<0$. As $v$ we can take a constant function $v=t$ with $t>0$ sufficiently large. We set

$$
c^{*}=\frac{1}{N} \min \left(\frac{S_{-\gamma}^{\frac{N}{2}}}{2 Q(0)^{\frac{N-2}{2}}}, \frac{S^{\frac{N}{2}}}{2 Q_{m^{\frac{N-2}{2}}}}, \frac{S^{\frac{N}{2}}}{Q_{M}^{\frac{N-2}{2}}}\right) .
$$


We commence with the case

I. $\quad c^{*}=\frac{S^{\frac{N}{2}}}{2 N Q_{m}^{\frac{N-2}{2}}}$.

To verify that $\tilde{c}<c^{*}$ we use the instanton $U(x)=\frac{d_{N}}{\left(1+|x|^{2}\right)^{\frac{N-2}{2}}}$, where $d_{N}>0$ is a constant. For a suitable choice of $d_{N}$, the function $U$ satisfies the equation

$$
-\Delta u=|u|^{2^{*}-2} u \quad \text { in } \quad \mathbb{R}^{N} .
$$

Moreover we have $\int_{\mathbb{R}^{N}} U^{2^{*}} d x=\int_{\mathbb{R}^{N}}|\nabla U|^{2} d x=S^{\frac{N}{2}}$. We set

$$
U_{\epsilon, y}(x)=\frac{d_{N} \epsilon^{\frac{N-2}{2}}}{\left(\epsilon^{2}+|x-y|^{2}\right)^{\frac{N-2}{2}}}, \quad y \in \mathbb{R}^{N}, \quad \epsilon>0 .
$$

We now check that for $y \neq 0$

$$
\int_{\Omega} \frac{1}{|x|^{2}} U_{\epsilon, y}(x)^{2} d x=\left\{\begin{array}{llc}
O\left(\epsilon^{2}\right) & \text { if } \quad N \geq 5 \\
O\left(\epsilon^{2} \log \frac{1}{\epsilon}\right) & \text { if } \quad N=4 \\
O(\epsilon) & \text { if } \quad N=3
\end{array}\right.
$$

Let $\rho>0$. We split the integration

$$
\begin{aligned}
\int_{\Omega} \frac{1}{|x|^{2}} U_{\epsilon, y}(x)^{2} d x= & d_{N}^{2} \int_{\Omega \cap|x-y| \leq \rho} \frac{\epsilon^{N-2}}{|x|^{2}\left(\epsilon^{2}+|y-x|^{2}\right)^{N-2}} d x \\
& +d_{N}^{2} \int_{\Omega \cap|x-y| \geq \rho} \frac{\epsilon^{N-2}}{|x|^{2}\left(\epsilon^{2}+|x-y|^{2}\right)^{N-2}} d x=J_{1}+J_{2} .
\end{aligned}
$$

It is clear that $J_{2}=O\left(\epsilon^{N-2}\right)$. To estimate $J_{1}$ we choose $\rho<|y|$. If $N \geq 5$, then letting $a=d_{N}^{2} \frac{1}{(|y|-\rho)^{2}}$ we get

$$
J_{1} \leq a \epsilon^{2} \int_{|x|<\frac{\rho}{\epsilon}} \frac{d x}{\left(1+|x|^{2}\right)^{N-2}} \leq a \epsilon^{2} \int_{\mathbb{R}^{N}} \frac{d x}{\left(1+|x|^{2}\right)^{N-2}}<\infty .
$$

If $N=4$, then

$$
J_{1}=a \epsilon^{2} \int_{|x|<\frac{\rho}{\epsilon}} \frac{1}{\left(1+|x|^{2}\right)^{2}} d x=C \epsilon^{2} \int_{0}^{\frac{\rho}{\epsilon}} \frac{r^{3}}{\left(1+r^{2}\right)^{2}} d r=O\left(\epsilon^{2} \log \frac{1}{\epsilon}\right) .
$$

Finally, in the case $N=3$ we have

$$
J_{1} \leq a \epsilon^{2} \int_{|x|<\frac{\rho}{\epsilon}} \frac{d x}{1+|x|^{2}}=\tilde{a} \epsilon^{2} \int_{0}^{\frac{\rho}{\epsilon}} \frac{r^{2}}{1+r^{2}} d r=O(\epsilon)
$$


where $\tilde{a}$ is a positive constant independent of $\epsilon$. We also need the estimate

$$
k_{1} \leq \int_{\Omega} \frac{1}{|x|^{2}} U_{\epsilon, 0}(x)^{2} d x \leq k_{2}
$$

for all $\epsilon>0$ and some constants $k_{1}, k_{2}>0$. We denote by $H(y)$ the mean curvature of $\partial \Omega$ at $y \in \partial \Omega$. We put

$$
E_{\lambda, \gamma}(u)=\frac{\int_{\Omega}\left(|\nabla u|^{2}+\frac{\lambda u^{2}}{|x-a|^{2}}-\frac{\gamma u^{2}}{|x|^{2}}\right) d x}{\left(\int_{\Omega}|u|^{2^{*}} d x\right)^{\frac{N-2}{N}}} .
$$

Suppose that $y \neq a, 0$, then

$$
E_{\lambda, \gamma}\left(U_{\epsilon, y}\right) \leq \frac{S}{2^{\frac{2}{N}}}- \begin{cases}A_{N} H(y) \epsilon \log \frac{1}{\epsilon}+O(\epsilon), & N=3, \\ A_{N} H(y) \epsilon+O\left(\epsilon^{2} \log \frac{1}{\epsilon}\right), & N=4, \\ A_{N} H(y) \epsilon+O\left(\epsilon^{2}\right), & N \geq 5,\end{cases}
$$

where $A_{N}>0$ is a constant depending on $N$. This asymptotic estimate has been established in the papers [31] and [1] with singular terms replaced by $u^{2}$. Combining the estimate from [31] (or [1]) with (19) we easily derive (20).

Theorem 2.1. Suppose that $c^{*}=\frac{S^{\frac{N}{2}}}{2 N Q_{m^{2}}^{\frac{N-2}{2}}}, Q_{m}=Q(y), y \neq a, 0$ and that

$$
|Q(x)-Q(y)|=o(|x-y|) \quad \text { for } x \text { near } y,
$$

with $H(y)>0$. If $\gamma<\gamma_{\circ}(\lambda)$, where $\gamma_{\circ}(\lambda)$ is a constant from Proposition 1.5, then problem (1) admits a positive solution.

Proof. Since $c^{*}=\frac{S^{\frac{N}{2}}}{2 N Q_{m}^{\frac{N-2}{2}}}$ and $S_{-\gamma}<S$ we see that $y \neq 0$. We derive from (21) the following expansion

$$
\int_{\Omega} Q(x) U_{\epsilon, y}^{2^{*}} d x=Q(y) \int_{\Omega} U_{\epsilon, y}^{2^{*}} d x+o(\epsilon) .
$$

Indeed, for every $\eta>0$ we can find $\delta(\eta)>0$ such that

$$
|Q(x)-Q(y)|<\eta|x-y| \text { for }|x-y|<\delta(\eta) .
$$

Then

$$
\begin{aligned}
\left|\int_{\Omega}(Q(x)-Q(y)) U_{\epsilon, y}^{2^{*}} d x\right| \leq & \eta \int_{\Omega \cap(|x-y|<\delta(\eta))}|x-y| U_{\epsilon, y}^{2^{*}} d x \\
& +2 Q_{M} \int_{\Omega \cap(|x-y| \geq \delta(\eta))} U_{\epsilon, y}^{2^{*}} d x \\
\leq & C\left(\eta \epsilon+\epsilon^{N} \int_{\delta(\eta)}^{\infty} \frac{r^{N-1}}{r^{2 N}} d r\right)=\tilde{C}\left(\epsilon \eta+\frac{\epsilon^{N}}{\delta(\eta)^{N}}\right),
\end{aligned}
$$


where $C$ and $\tilde{C}$ are positive constants independent of $\eta$ and $\epsilon$. From this we derive that

$$
\limsup _{\epsilon \rightarrow 0} \frac{1}{\epsilon} \int_{\Omega}|Q(x)-Q(y)| U_{\epsilon, y}^{2^{*}} d x \leq \tilde{C} \eta .
$$

Since $\eta>0$ is arbitrary, this limit is equal to 0 and (22) follows. It then follows from (20) and (22) that

$$
\begin{aligned}
\sup _{t \geq 0} J_{\lambda, \gamma}\left(t U_{\epsilon, y}\right) & =\frac{1}{N} \frac{\left(\int_{\Omega}\left(\left|\nabla U_{\epsilon, y}\right|^{2}+\frac{\lambda U_{\epsilon, y}^{2}}{|x-a|^{2}}-\frac{\gamma U_{\epsilon, y}^{2}}{|x|^{2}}\right) d x\right)^{\frac{N}{2}}}{\left(\int_{\Omega} Q(y) U_{\epsilon, y}^{2^{*}} d x+o(\epsilon)\right)^{\frac{N-2}{2}}} \\
& <\frac{S^{\frac{N}{2}}}{2 N Q_{m^{\frac{N-2}{2}}}^{2}}
\end{aligned}
$$

for $\epsilon>0$ sufficiently small.

We now consider the case

$$
\text { II. } \quad c^{*}=\frac{S_{-\gamma}^{\frac{N}{2}}}{2 N Q(0)^{\frac{N-2}{2}}} \text {. }
$$

To show that the mountain-pass level satisfies $\tilde{c}<c^{*}$ we use the function $V_{\gamma, \epsilon}$ which satisfies the equation (8). Since $a \neq 0$, we have the following asymptotic estimate

$$
\int_{\Omega} \frac{V_{\gamma, \epsilon}(x)^{2}}{|x-a|^{2}} d x \leq\left\{\begin{array}{lll}
C \epsilon^{2 \frac{\sqrt{\mu}}{\sqrt{\mu}-\gamma}} & \text { if } & 0<\gamma<\bar{\mu}-1 \\
C \epsilon^{2 \sqrt{\mu}}|\log \epsilon| & \text { if } & \gamma=\bar{\mu}-1
\end{array}\right.
$$

To show this we take $0<\rho<|a|$ and write

$$
\begin{aligned}
\int_{\Omega} \frac{V_{\gamma, \epsilon}(x)^{2}}{|x-a|^{2}} d x & =\int_{\Omega \cap(|x|<\rho)} \frac{V_{\gamma, \epsilon}(x)^{2}}{|x-a|^{2}} d x+\int_{\Omega \cap(|x| \geq \rho)} \frac{V_{\gamma, \epsilon}(x)^{2}}{|x-a|^{2}} d x \\
& \leq \int_{|x|<\rho} \frac{V_{\gamma, \epsilon}(x)^{2}}{|x-a|^{2}} d x+O\left(\epsilon^{N-2}\right)=I+O\left(\epsilon^{N-2}\right) .
\end{aligned}
$$

Changing variables $x=y \epsilon^{\frac{\sqrt{\mu}}{\sqrt{\mu-\gamma}}}$ in the integral $I$ we get

$$
I=C \int_{|x|<\frac{\rho}{\epsilon \frac{\sqrt{\bar{\mu}}}{\sqrt{\bar{\mu}-\gamma}}}} \frac{\epsilon^{2 \frac{\sqrt{\mu}}{\sqrt{\bar{\mu}-\gamma}}}}{\left(|x|^{\frac{\gamma^{\prime}}{\sqrt{\mu}}}+|x|^{\frac{\gamma}{\sqrt{\mu}}}\right)^{2 \sqrt{\bar{\mu}}}} d x .
$$

If $\bar{\mu}<\gamma-1$, then

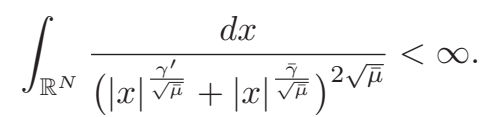


On the other hand if $\gamma=\bar{\mu}-1$, then

$$
\int_{|x|<\frac{\rho}{\epsilon}} \frac{d x}{\left(|x|^{\frac{\gamma^{\prime}}{\sqrt{\mu}}}+|x|^{\frac{\bar{\gamma}}{\sqrt{\mu}}}\right)^{2 \sqrt{\bar{\mu}}}}=\int_{|x|<\frac{\rho}{\epsilon}} \frac{d x}{\left(|x|^{\frac{\sqrt{\bar{\mu}}-1}{\sqrt{\mu}}}+|x|^{\frac{\sqrt{\bar{\mu}}+1}{\sqrt{\mu}}}\right)^{2 \sqrt{\mu}}}=O\left(\log \frac{1}{\epsilon}\right) .
$$

We now recall the following asymptotic relations from paper [23]:

$$
\begin{gathered}
\int_{\Omega}\left|\nabla V_{\gamma, \epsilon}\right|^{2} d x=\int_{\mathbb{R}_{+}^{N}}\left|\nabla V_{\gamma, \epsilon}\right|^{2} d x-I_{1}(\epsilon)+o\left(\epsilon^{\frac{\sqrt{\mu}}{\sqrt{\mu}-\gamma}}\right)+O\left(\epsilon^{N-2}\right), \\
\int_{\Omega} \frac{V_{\gamma, \epsilon}^{2}}{|x|^{2}} d x=\int_{\mathbb{R}_{+}^{N}} \frac{V_{\gamma, \epsilon}^{2}}{|x|^{2}} d x-I_{2}(\epsilon)+o\left(\epsilon^{\frac{\sqrt{\bar{\mu}}}{\sqrt{\mu}-\gamma}}\right)+O\left(\epsilon^{N-2}\right), \\
\int_{\Omega} V_{\gamma, \epsilon}^{2^{*}} d x=\int_{\mathbb{R}_{+}^{N}} V_{\gamma, \epsilon}^{2^{*}} d x-I_{3}(\epsilon)+o\left(\epsilon^{\frac{\sqrt{\mu}}{\sqrt{\bar{\mu}}-\gamma}}\right)+O\left(\epsilon^{N}\right),
\end{gathered}
$$

where $I_{1}(\epsilon), I_{2}(\epsilon)$ and $I_{3}(\epsilon)$ satisfy, respectively,

$$
\begin{aligned}
& \lim _{\epsilon \rightarrow 0} \epsilon^{-\frac{\sqrt{\mu}}{\sqrt{\bar{\mu}-\gamma}}} I_{1}(\epsilon)=I_{1}:=\int_{\mathbb{R}^{N-1}} \frac{\left(\gamma^{\prime}\left|y^{\prime}\right|^{\frac{\gamma^{\prime}}{\sqrt{\mu}}}+\gamma\left|y^{\prime}\right|^{\frac{\bar{\gamma}}{\bar{\mu}}}\right)^{2} g\left(y^{\prime}\right)}{\left|y^{\prime}\right|^{2}\left(\left|y^{\prime}\right|^{\frac{\gamma^{\prime}}{\sqrt{\mu}}}+\left|y^{\prime}\right|^{\frac{\bar{\gamma}}{\sqrt{\mu}}}\right)^{2(\sqrt{\bar{\mu}}+1)} d y^{\prime}>0,} \\
& \lim _{\epsilon \rightarrow 0} \epsilon^{-\frac{\sqrt{\bar{\mu}}}{\sqrt{\bar{\mu}-\gamma}}} I_{2}(\epsilon)=I_{2}:=\int_{\mathbb{R}^{N-1}} \frac{g\left(y^{\prime}\right)}{\left|y^{\prime}\right|^{2}\left(\left|y^{\prime}\right|^{\frac{\gamma^{\prime}}{\sqrt{\mu}}}+\left|y^{\prime}\right|^{\frac{\bar{\gamma}}{\sqrt{\mu}}}\right)^{2 \sqrt{\bar{\mu}}}} d y^{\prime}>0, \\
& \lim _{\epsilon \rightarrow 0} \epsilon^{-\frac{\sqrt{\bar{\mu}}}{\sqrt{\bar{\mu}-\gamma}}} I_{3}(\epsilon)=I_{3}:=\int_{\mathbb{R}^{N-1}} \frac{g\left(y^{\prime}\right)}{\left(\left|y^{\prime}\right|^{\frac{\gamma^{\prime}}{\sqrt{\mu}}}+\left|y^{\prime}\right|^{\frac{\bar{\gamma}}{\sqrt{\mu}}}\right)^{N}} d y^{\prime}>0,
\end{aligned}
$$

and $g\left(y^{\prime}\right)=\frac{1}{2} \sum_{i=1}^{N-1} \lambda_{i} y_{i}$. Here $\lambda_{i}$ denote the principal curvatures of $\partial \Omega$ at 0 . Then it follows from the above asymptotic relations and (23) that

$$
E_{\lambda, \gamma}\left(V_{\gamma, \epsilon}\right)<\frac{S_{-\gamma}}{2^{\frac{2}{N}}}-C_{1} \epsilon^{\frac{\sqrt{\bar{\mu}}}{\sqrt{\bar{\mu}-\gamma}}}+o\left(\epsilon^{\frac{\sqrt{\bar{\mu}}}{\sqrt{\bar{\mu}-\gamma}}}\right)
$$

for $N \geq 5, \gamma<\gamma_{\circ}(\lambda), \gamma<\bar{\mu}-1$, and $\epsilon>0$ sufficiently small, where $H(0)>0$ and $C_{1}>0$ is a constant independent of $\epsilon$. For the computational details we refer to paper [23].

Theorem 2.2. Suppose that $c^{*}=\frac{S_{-\gamma}^{\frac{N}{2}}}{2 N Q(0)^{\frac{N-2}{2}}}, \gamma<\gamma_{\circ}(\lambda), N \geq 5$, and $\gamma<\bar{\mu}-1$. Moreover assume that

$$
|Q(x)-Q(0)|=o(|x|) \quad \text { for } x \text { close to } 0
$$

with $H(0)>0$. Then problem (1) admits a positive solution. 
Proof. This follows from (24) and the following formula

$$
\int_{\Omega} Q(x) V_{\gamma, \epsilon}^{2^{*}} d x=Q(0) \int_{\Omega} V_{\gamma, \epsilon}^{2^{*}} d x+o\left(\epsilon^{\frac{\sqrt{\bar{\mu}}}{\sqrt{\bar{\mu}-\gamma}}}\right) .
$$

We now prove this expansion. Given $\eta>0$ we choose $\delta(\eta)>0$ so that

$$
|Q(x)-Q(0)|<\eta|x| \quad \text { for }|x|<\delta(\eta) \text {. }
$$

Then

$$
\begin{aligned}
\int_{\Omega}|Q(x)-Q(0)| V_{\gamma, \epsilon}^{2^{*}} d x & \leq \eta \int_{\Omega \cap(|x|<\delta(\eta))}|x| V_{\gamma, \epsilon}^{2^{*}} d x+2 Q_{M} \int_{\Omega \cap(|x| \geq \delta(\eta))} V_{\gamma, \epsilon}^{2^{*}} d x \\
& =J_{1}+J_{2} .
\end{aligned}
$$

Using the change of variables $x=y \epsilon^{\frac{\sqrt{\mu}}{\sqrt{\bar{\mu}-\gamma}}}$ we get

$$
\begin{aligned}
J_{1} & \leq \epsilon^{N} \eta \int_{\mathbb{R}^{N}} \frac{|x|}{\left(\epsilon^{2}|x|^{\frac{\gamma^{\prime}}{\sqrt{\mu}}}+|x|^{\frac{\gamma}{\sqrt{\mu}}}\right)^{N}} d x \\
& =C \eta \epsilon^{\frac{\sqrt{\mu}}{\sqrt{\mu}-\gamma}} \int_{\mathbb{R}^{N}} \frac{|y|}{\left(|y|^{\frac{\gamma^{\prime}}{\sqrt{\mu}}}+|y|^{\frac{\bar{\gamma}}{\sqrt{\mu}}}\right)^{N}} d y=C_{1} \eta \epsilon^{\frac{\sqrt{\mu}}{\sqrt{\mu}-\gamma}},
\end{aligned}
$$

where $C$ and $C_{1}$ are positive constants independent of $\epsilon$ and $\eta$. For $J_{2}$ we have the estimate

$$
J_{2} \leq 2 Q_{M} C \int_{\delta(\eta)}^{\infty} \frac{\epsilon^{N} r^{N-1}}{r^{\frac{\bar{\gamma} N}{\sqrt{\mu}}}} d r=O\left(\epsilon^{N}\right) .
$$

Since $\eta$ is arbitrary and $\frac{\sqrt{\bar{\mu}}}{\sqrt{\bar{\mu}-\gamma}}<N$, provided $\gamma<\bar{\mu}-1$, our expansion follows.

Finally, we consider the case

$$
\text { III. } \quad c^{*}=\frac{S^{\frac{N}{2}}}{N Q_{M}^{\frac{N-2}{2}}} \text {. }
$$

In this case it is convenient to seek a solution of problem (1) as a minimizer of the constrained minimization problem

$$
S_{\lambda, \gamma}=\inf \left\{\int_{\Omega}\left(|\nabla u|^{2}+\frac{\lambda u^{2}}{|x-a|^{2}}-\frac{\gamma u^{2}}{|x|^{2}}\right) d x ; u \in H^{1}(\Omega), \int_{\Omega} Q(x)|u|^{2^{*}} d x=1\right\} .
$$

We recall the existence theorem (see [11]) for the critical problem

$$
\begin{cases}-\Delta u+\mu u & =Q(x)|u|^{2^{*}-2} u \text { in } \Omega \\ \frac{\partial u}{\partial \nu} & =0 \text { on } \partial \Omega .\end{cases}
$$


Theorem 2.3. Suppose that $Q_{M}>2^{\frac{2}{N-2}} Q_{m}$ and $N \geq 5$. Then there exists a constant $K>0$ such that for $0<\mu \leq K$ problem (25) has a positive solution (called a least energy solution) and no least energy solution for $\mu>K$. Moreover, we have for $\mu \geq K$

$$
\frac{S}{Q_{M}^{\frac{N-2}{N}}}=\inf _{u \in H^{1}(\Omega)-\{0\}} \frac{\int_{\Omega}\left(|\nabla u|^{2}+\mu u^{2}\right) d x}{\left(\int_{\Omega} Q(x)|u|^{2^{*}} d x\right)^{\frac{2}{2^{*}}}} .
$$

It follows from the proof of Proposition 1.5 that $S_{\lambda, \gamma}>0$ for $0<\gamma<\gamma_{\circ}(\lambda)$. According to Proposition 1.4 there exists a minimizer for $S_{\lambda, \gamma}$ if

$$
S_{\lambda, \gamma}<\min \left(\frac{S}{2^{\frac{2}{N}} Q^{\frac{N-2}{N}}}, \frac{S_{-\gamma}}{2^{\frac{2}{N}} Q(0)^{\frac{N-2}{N}}}, \frac{S}{Q_{M}^{\frac{N-2}{N}}}\right) .
$$

First with the aid of Theorem 2.3 we deduce the existence result for problem (1) with $\gamma=0$, that is,

$$
\begin{cases}-\Delta u+\frac{\lambda}{|x-a|^{2}} u & =Q(x)|u|^{2^{*}-2} u \text { in } \Omega \\ \frac{\partial u}{\partial \nu} & =0 \text { on } \partial \Omega\end{cases}
$$

Theorem 2.4. Suppose that $Q_{M}>2^{\frac{2}{N-2}} Q_{m}$ and $N \geq 5$. Then there exists a constant $K_{1}>0$ such that for $0<\lambda \leq K_{1}$ problem (26) admits a solution ( $a$ least energy solution) and no least energy solution for $\lambda>K_{1}$. Moreover we have $S_{\lambda, 0}=\frac{S}{Q_{M}^{\frac{N-2}{N}}}$ for $\lambda \geq K_{1}$.

This follows from the estimate

$$
\int_{\Omega}\left(|\nabla u|^{2}+\frac{\lambda}{|x-a|^{2}} u^{2}\right) d x \geq \int_{\Omega}\left(|\nabla u|^{2}+\frac{\lambda}{d^{2}} u^{2}\right) d x
$$

where $d=\operatorname{diam} \Omega$.

We are now in a position to formulate the existence result for problem (1).

Theorem 2.5. Suppose that $c^{*}=\frac{S^{\frac{N}{2}}}{N Q^{\frac{N-2}{M^{2}}}}$ and $N \geq 5$ and let $\gamma_{\circ}(\lambda)$ be a constant from Proposition 1.5. If $\gamma<\gamma_{\circ}$ and $\lambda<K_{1}$, then problem (1) has a solution which is multiple of a minimizer for $S_{\lambda, \gamma}$.

Proof. Let $\lambda<K_{1}$ and let $u_{\lambda}$ be a minimizer for $S_{\lambda, 0}$ with $\int_{\Omega} Q(x)\left|u_{\lambda}\right|^{2^{*}} d x=1$. We then have

$$
\int_{\Omega}\left(\left|\nabla u_{\lambda}\right|^{2}+\frac{\lambda u_{\lambda}^{2}}{|x-a|^{2}}-\frac{\gamma u_{\lambda}^{2}}{|x|^{2}}\right) d x=\frac{S}{Q_{M}^{\frac{N-2}{N}}}-\gamma \int_{\Omega} \frac{u_{\lambda}^{2}}{|x|^{2}} d x
$$

This shows that $S_{\lambda, \gamma}<\frac{S}{Q_{M}^{\frac{N-2}{N}}}$ and the result follows. 


\section{Regularity of solutions}

First we consider problem (1) with $\gamma=0$.

Proposition 3.1. Suppose $\gamma=0$. Then solutions of (1) belong to $L^{\infty}(\Omega)$.

This follows from the proof of Theorem 3.4 below.

We now consider solutions of problem (1) with $\gamma>0$. We now prove a Brézis-Kato type result for solutions of problem (1) (see also Theorem 2.3 in [28]).

Proposition 3.2. Let $d_{1}=\min \left(1, \frac{\lambda}{d^{2}}\right)$ and let $S_{1}$ be the best Sobolev constant for the embedding of $H^{1}(\Omega)$ into $L^{2^{*}}(\Omega)$. If $p$ satisfies

$$
\gamma(p+2)\left(\frac{1}{\bar{\mu}}+1\right)<4 d_{1} S_{1},
$$

then a solution $u$ of (1) belongs to $L^{p}(\Omega)$. (If $\gamma>0$ is small enough we can ensure that $p>2^{*}$.)

Proof. We apply the Moser iteration technique. As a test function we take

$$
\phi=u \min (u, L)^{p-2}=u u_{L}^{p-2},
$$

where $u_{L}=\min (u, L), L>0$ and $p>2$. We obtain

$$
\begin{aligned}
& \int_{\Omega}|\nabla u|^{2} u_{L}^{p-2} d x+(p-2) \int_{\Omega} \nabla u \nabla u_{L} u_{L}^{p-3} u d x+\lambda \int_{\Omega} \frac{u^{2} u_{L}^{p-2}}{|x-a|^{2}} d x \\
&=\int_{\Omega} Q(x)|u|^{2^{*}-2} u^{2} u_{L}^{p-2} d x+\gamma \int_{\Omega} \frac{u^{2} u_{L}^{p-2}}{|x|^{2}} d x .
\end{aligned}
$$

From this we deduce

$$
\begin{aligned}
\int_{\Omega}|\nabla u|^{2} u_{L}^{p-2} d x+(p-2) \int_{\Omega}\left|\nabla u_{L}\right|^{2} u_{L}^{p-2} d x+\frac{\lambda}{d^{2}} \int_{\Omega} u^{2} u_{L}^{p-2} d x \\
\quad \leq Q_{M} \int_{\Omega} u^{2^{*}-2} u^{2} u_{L}^{p-2} d x+\gamma \int_{\Omega} \frac{u^{2} u_{L}^{p-2}}{|x|^{2}} d x .
\end{aligned}
$$

On the other hand we have

$$
\begin{aligned}
\int_{\Omega}\left|\nabla\left(u u_{L}^{\frac{p}{2}-1}\right)\right|^{2} d x= & \int_{\Omega}|\nabla u|^{2} u_{L}^{p-2} d x+\frac{(p-2)^{2}}{4} \int_{\Omega}\left|\nabla u_{L}\right|^{2} u_{L}^{p-4} u^{2} d x \\
& +(p-2) \int_{\Omega} \nabla u \nabla u_{L} u_{L}^{p-3} u d x \\
= & \int_{\Omega}|\nabla u|^{2} u_{L}^{p-2} d x+\frac{p^{2}-4}{4} \int_{\Omega}\left|\nabla u_{L}\right|^{2} u_{L}^{p-2} d x .
\end{aligned}
$$


From (28) we deduce

$$
\begin{aligned}
\int_{\Omega}|\nabla u|^{2} u_{L}^{p-2} d x+\frac{\left(p^{2}-4\right)}{4} \int_{\Omega}\left|\nabla u_{L}\right|^{2} u_{L}^{p-2} d x+\frac{\lambda}{d^{2}} \int_{\Omega} u^{2} u_{L}^{p-2} d x \\
\leq \frac{p+2}{4} Q_{M} \int_{\Omega}|u|^{2^{*}-2} u^{2} u_{L}^{p-2} d x+\frac{p+2}{4} \gamma \int_{\Omega} \frac{u^{2} u_{L}^{p-2}}{|x|^{2}} d x .
\end{aligned}
$$

This, in conjunction with (29), gives

$$
\begin{aligned}
d_{1} \int_{\Omega}\left(\left|\nabla\left(u u_{L}^{\frac{p}{2}-1}\right)\right|^{2}+\left(u u_{L}^{\frac{p}{2}-1}\right)^{2}\right) d x & \\
& \leq \frac{p+2}{4} Q_{M} \int_{\Omega} u^{2^{*}-2} u^{2} u_{L}^{p-2} d x+\frac{p+2}{4} \gamma \int_{\Omega} \frac{u^{2} u_{L}^{p-2}}{|x|^{2}} d x .
\end{aligned}
$$

We now apply Lemma 1.1 with $\epsilon=1$ and choose $\gamma>0$ so that

$$
\frac{p+2}{4} \gamma\left(\frac{1}{\bar{\mu}}+1\right)<d_{1}
$$

and a constant $K_{1}>0$ such that

$$
\frac{p+2}{4} Q_{M}\left(\int_{u \geq K_{1}} u^{\frac{N\left(2^{*}-2\right)}{2}} d x\right)^{\frac{2}{N}} \leq \frac{d_{2} S}{2},
$$

where $d_{2}=d_{1}-\frac{p+2}{4} \gamma\left(\frac{1}{\bar{\mu}}+1\right)$. With the aid of the Sobolev inequality we obtain that

$$
\begin{aligned}
\frac{S_{1} d_{2}}{2}\left(\int_{\Omega}\left(u u_{L}^{\frac{p}{2}-1}\right)^{2^{*}}\right. & d x)^{\frac{2}{2^{*}}} \\
& \leq \frac{p+2}{4} Q_{M} K_{1}^{2^{*}-2} \int_{\Omega} u^{2} u_{L}^{p-2} d x+\frac{p+2}{4} \gamma C(1) \int_{\Omega} u^{2} u_{L}^{p-2} d x .
\end{aligned}
$$

Letting $L \rightarrow \infty$ and then iterating the resulting inequality, starting with $p=2^{*}$, the assertion follows as long as $\gamma(p+2)\left(\frac{1}{\bar{\mu}}+1\right)<4 d_{1} S_{1}$.

By the regularity theory for elliptic equations solutions of (1) are in $C^{1}(\bar{\Omega}-\{a, 0\})$. We now examine the behavior of solutions around points $a$ and 0 . We need a version of the Caffarelli-Kohn-Nirenberg inequality [8,9]. We recall that if $0 \in \Omega$, then for every $u \in H_{\circ}^{1}\left(\Omega,|x|^{-2 \alpha} d x\right)$ we have

$$
\int_{\Omega}|x|^{-2 \alpha}|\nabla u|^{2} d x \geq C_{\alpha, \beta}^{-1}\left(\int_{\Omega}|x|^{-\beta p}|u|^{p} d x\right)^{\frac{2}{p}},
$$


where $-\infty<\alpha<\frac{N-2}{2}, \alpha \leq \beta \leq \alpha+1, p=\frac{2 N}{N-2+2(\beta-\alpha)}$ and a constant $C_{\alpha, \beta}>0$ depends on $\alpha$ and $\beta$. Let $\tilde{H}^{1}(\Omega):=H^{1}\left(\Omega,|x|^{-2 \alpha} d x\right)$ be the Sobolev space equipped with the norm

$$
\|u\|_{\tilde{H}^{1}}^{2}=\int_{\Omega}|x|^{-2 \alpha}|\nabla u|^{2} d x+\int_{\Omega} u^{2} d x .
$$

We need the following version of the above inequality.

Lemma 3.3. Let $0 \in \partial \Omega$. Then for every $\epsilon>0$ there exists a constant $C(\epsilon)>0$ such that

$$
\begin{aligned}
& \left(\int_{\Omega}|x|^{-\beta p}|u|^{p} d x\right)^{\frac{2}{p}} \\
& \quad \leq\left(C_{\alpha, \beta}+\epsilon\right) \int_{\Omega}|x|^{-2 \alpha}|\nabla u|^{2} d x+C(\epsilon) \int_{\Omega} u^{2} d x+C(\epsilon)\left(\int_{\Omega}|u|^{p} d x\right)^{\frac{2}{p}}
\end{aligned}
$$

for every $u \in \tilde{H}^{1}(\Omega)$.

Proof. The proof is similar to that of Lemma 1.1. With notations from the proof of this lemma we first consider the case $\psi \equiv 0$. Let $u \in \tilde{H}^{1}\left(B\left(0, \delta_{\circ}\right)\right)$ with supp $u \subset$ $B\left(0, \delta_{\circ}\right)$. Since the values of $u$ for $x_{N}<0$ are irrelevant, we may assume that $u$ is an even function in $x_{N}$. Then

$$
\begin{aligned}
C_{\alpha, \beta} \int_{A}|x|^{-2 \alpha}|\nabla u|^{2} d x & =\frac{C_{\alpha, \beta}}{2} \int_{B\left(0, \delta_{\circ}\right)}|x|^{-2 \alpha}|\nabla u|^{2} d x \\
& \geq \frac{1}{2}\left(\int_{B\left(0, \delta_{\circ}\right)}|x|^{-2 \beta p}|u|^{p} d x\right)^{\frac{2}{p}}=2^{\frac{2}{p}-1}\left(\int_{A} \frac{|u|^{p}}{|x|^{\beta p}} d x\right)^{\frac{2}{p}} .
\end{aligned}
$$

In the next step of the proof we use the mapping from the proof of Lemma 1.1 that straightens the boundary $\partial \Omega$ around 0 to obtain a local version of our inequality. Finally, let $\phi$ be a function in $C_{\circ}^{1}(B(0, \delta))$ such that $\phi(x)=1$ for $x$ near 0 and $0 \leq \phi(x) \leq 1$ on $B(0, \delta)$. By the first part of the proof we have

$$
\begin{aligned}
\left(\int_{\Omega} \frac{|u|^{p}}{|x|^{p \beta}} d x\right)^{\frac{2}{p}} & \leq 2^{\frac{2}{p}-1}\left(\int_{\Omega} \frac{|u \phi|^{p}}{|x|^{p \beta}} d x\right)^{\frac{2}{p}}+2^{\frac{2}{p}-1}\left(\int_{\Omega} \frac{|u|^{p}\left(1-\phi^{p}\right)}{|x|^{p \beta}} d x\right)^{\frac{2}{p}} \\
& \leq\left(C_{\alpha \beta}+2^{\frac{2}{p}-1} \epsilon\right) \int_{\Omega}|x|^{-2 \alpha}|\nabla(u \phi)|^{2} d x+2^{\frac{2}{p}-1} C(\delta)\left(\int_{\Omega}|u|^{p} d x\right)^{\frac{2}{p}} .
\end{aligned}
$$

Using the Young inequality we derive from this (30).

Inequality (30) can be reformulated, with the aid of Sobolev inequality, in the following way: there exists a constant $C_{1}>0$ such that

$$
\left(\int_{\Omega} \frac{|u|^{p}}{|x|^{p \beta}} d x\right)^{\frac{2}{p}} \leq C_{1}\left(\int_{\Omega}|x|^{-2 \alpha}|\nabla u|^{2} d x+\int_{\Omega} u^{2} d x\right)
$$


for every $u \in \tilde{H}^{1}(\Omega)$. This form of the CKN inequality will be used in the proof of Theorem 3.4 below.

Theorem 3.4. Let $N \geq 3$. Then for $\gamma \leq \gamma_{\circ}$ any solution $u$ of (1) satisfies

$$
|u(x)| \leq C|x|^{-(\sqrt{\bar{\mu}}-\sqrt{\bar{\mu}-\gamma})} \quad \text { for } x \in \Omega-\{0\} .
$$

Proof. We follow the ideas from the paper [6] which are modifications of the Moser iteration technique. We put $v(x)=|x|^{(\sqrt{\bar{\mu}}-\sqrt{\bar{\mu}-\gamma})} u(x)$. By straightforward computations we verify that

$$
\begin{aligned}
-\operatorname{div}\left(|x|^{-2(\sqrt{\bar{\mu}}-\sqrt{\bar{\mu}-\gamma})}\right. & \nabla v) \\
& =Q(x)|x|^{-2^{*}(\sqrt{\mu}-\sqrt{\bar{\mu}-\gamma})}|v|^{2^{*}-2} v-\lambda \frac{v}{|x|^{2(\sqrt{\bar{\mu}}-\sqrt{\bar{\mu}-\gamma})}|x-a|^{2}} .
\end{aligned}
$$

We take as a test function $\phi=\eta^{2} v v_{L}^{2(s-1)}$, where $\eta \in C_{\mathrm{o}}^{1}(B(0, \rho))$ with $\eta=1$ on $B(0, r), r<\rho,|\nabla \eta| \leq \frac{4}{\rho-r}$. Here $s>1$ and $L>0$ are constants. We then get

$$
\begin{aligned}
\int_{\Omega}|x|^{-2(\sqrt{\mu}-\sqrt{\bar{\mu}-\gamma})}\left[\eta^{2}|\nabla v|^{2}\right. & v_{L}^{2(s-1)} \\
& \left.+2(s-1) \eta^{2} v_{L}^{2(s-1)}\left|\nabla v_{L}\right|^{2}+2 \eta \nabla \eta \nabla v v v_{L}^{2(s-1)}\right] d x \\
= & \int_{\Omega} Q(x)|x|^{-2^{*}(\sqrt{\bar{\mu}}-\sqrt{\bar{\mu}-\gamma})} \eta^{2} v^{2^{*}} v_{L}^{2(s-1)} d x \\
& -\lambda \int_{\Omega}|x|^{-2(\sqrt{\bar{\mu}}-\sqrt{\bar{\mu}-\gamma})}|x-a|^{-2} \eta^{2} v^{2} v_{L}^{2(s-1)} d x .
\end{aligned}
$$

By the Young inequality for every $\epsilon>0$ there exists $C(\epsilon)>0$ such that

$$
\begin{aligned}
2 \int_{\Omega}|x|^{-2(\sqrt{\bar{\mu}}-\sqrt{\bar{\mu}-\gamma})} \nabla \eta \nabla v \eta v v_{L}^{2(s-1)} & d x \leq \epsilon \int_{\Omega}|x|^{-2(\sqrt{\bar{\mu}}-\sqrt{\bar{\mu}-\gamma})} \eta^{2}|\nabla v|^{2} v_{L}^{2(s-1)} d x \\
& +C(\epsilon) \int_{\Omega}|x|^{-2(\sqrt{\bar{\mu}}-\sqrt{\bar{\mu}-\gamma})}|\nabla \eta|^{2} v^{2} v_{L}^{2(s-1)} d x .
\end{aligned}
$$

Applying this inequality with $\epsilon=\frac{1}{2}$ we derive from (32) that

$$
\begin{aligned}
\int_{\Omega}|x|^{-2(\sqrt{\bar{\mu}}-\sqrt{\bar{\mu}}-\sqrt{\bar{\mu}-\gamma})}\left[\eta^{2}|\nabla v|^{2} v_{L}^{2(s-1)}\right. & \left.+4(s-1) \eta^{2}\left|\nabla v_{L}\right|^{2} v_{L}^{2(s-1)}\right] d x \\
\leq & 2 Q_{M} \int_{\Omega}|x|^{-2^{*}(\sqrt{\bar{\mu}}-\sqrt{\bar{\mu}-\gamma})} \eta^{2} v_{L}^{2(s-1)} v^{2^{*}} d x \\
& +2 C\left(\frac{1}{2}\right) \int_{\Omega}|x|^{-2(\sqrt{\bar{\mu}}-\sqrt{\bar{\mu}-\gamma})}|\nabla \eta|^{2} v^{2} v_{L}^{2(s-1)} d x \\
& -\lambda \int_{\Omega}|x|^{-2(\sqrt{\bar{\mu}}-\sqrt{\bar{\mu}-\gamma})}|x-a|^{-2} v^{2} \eta^{2} v_{L}^{2(s-1)} d x .
\end{aligned}
$$


We now rewrite this inequality in the form

$$
\begin{aligned}
\int_{\Omega}|x|^{-2(\sqrt{\bar{\mu}}-\sqrt{\bar{\mu}-\gamma})}\left[\eta^{2}|\nabla v|^{2} v_{L}^{2(s-1)}\right. & +4(s-1) \eta^{2} v_{L}^{2(s-1)}\left|\nabla v_{L}\right|^{2} \\
& \left.+\lambda \eta^{2}|x-a|^{-2}|x|^{-2(\sqrt{\bar{\mu}}-\sqrt{\bar{\mu}-\gamma})} v^{2} v_{L}^{2(s-1)} t\right] d x \\
\leq & C\left[\int_{\Omega}|x|^{-2^{*}(\sqrt{\bar{\mu}}-\sqrt{\bar{\mu}-\gamma})} \eta^{2} v^{2^{*}} v_{L}^{2(s-1)} d x\right. \\
& \left.+\int_{\Omega}|x|^{-2(\sqrt{\bar{\mu}}-\sqrt{\bar{\mu}-\gamma})}|\nabla \eta|^{2} v^{2} v_{L}^{2(s-1)}\right] d x
\end{aligned}
$$

for some constant $C>0$ depending on $Q_{M}$. By choosing $\gamma_{\circ}$ smaller, if necessary, by virtue of Proposition 3.2 we may assume that $u \in L^{\left(2^{*}-2\right) q}(\Omega)$ with $2<\frac{2 q}{q-1}<2^{*}$. This guarantees that $\frac{N}{2}<q$. We now use this choice of $q$ to estimate the integral involving the exponent $2^{*}$. By the Hölder and Young inequalities for every $\epsilon>0$ we have

$$
\begin{aligned}
& \int_{\Omega}|x|^{-2^{*}(\sqrt{\bar{\mu}}-\sqrt{\bar{\mu}-\gamma})} \eta^{2} v^{2^{*}} v_{L}^{2(s-1)} d x \\
& =\int_{\Omega}|x|^{-2(\sqrt{\bar{\mu}}-\sqrt{\bar{\mu}-\gamma})}|u|^{2^{*}-2}\left|\eta v v_{L}^{(s-1)}\right|^{2} d x \\
& \leq\|u\|_{L^{\left(2^{*}-2\right) q}(\Omega)}^{\left(2^{*}-2\right)}\left\||\cdot|^{-(\sqrt{\bar{\mu}}-\sqrt{\bar{\mu}-\gamma})} \eta v v_{L}^{(s-1)}\right\|_{L^{\frac{2 q}{q-1}}(\Omega)}^{2} \\
& \leq\|u\|_{L^{\left(2^{*}-2\right) q}(\Omega)}^{2^{*}-2}\left(\epsilon\left\||\cdot|^{-(\sqrt{\bar{\mu}}-\sqrt{\bar{\mu}-\gamma})} \eta v v_{L}^{s-1}\right\|_{L^{2^{*}}(\Omega)}\right. \\
& \left.+C(n, q) \epsilon^{-\frac{N}{2 q-N}}\left\||\cdot|^{-(\sqrt{\bar{\mu}}-\sqrt{\bar{\mu}-\gamma})} \eta v v_{L}^{s-1}\right\|_{L^{2}(\Omega)}\right)^{2} \\
& \leq C \epsilon^{2}\left(\int_{\Omega}|x|^{-2^{*}(\sqrt{\mu}-\sqrt{\bar{\mu}-\gamma})}\left|\eta v v_{L}^{s-1}\right|^{2^{*}} d x\right)^{\frac{2}{2^{*}}} \\
& +C \epsilon^{-\frac{2 N}{2 q-N}} \int_{\Omega}|x|^{-(\sqrt{\bar{\mu}}-\sqrt{\bar{\mu}-\gamma})}\left|\eta v v_{L}^{s-1}\right|^{2} d x .
\end{aligned}
$$

The above inequality combined with (33) and (31) with $\alpha=\beta=\sqrt{\bar{\mu}}-\sqrt{\bar{\mu}-\gamma}<\frac{N-2}{2}$ 
gives

$$
\begin{aligned}
\left(\int_{\Omega}|x|^{-2^{*}(\sqrt{\bar{\mu}}-\sqrt{\bar{\mu}-\gamma})}\left|\eta v v_{L}^{s-1}\right|^{2^{*}} d x\right)^{\frac{2}{2^{*}}} & \\
\leq & C s \epsilon^{2}\left(\int_{\Omega}|x|^{-2^{*}(\sqrt{\bar{\mu}}-\sqrt{\bar{\mu}-\gamma})}\left|\eta v v_{L}^{s-1}\right|^{2^{*}} d x\right)^{\frac{2}{2^{*}}} \\
& +C s \int_{\Omega}|x|^{-2(\sqrt{\bar{\mu}}-\sqrt{\bar{\mu}-\gamma})}\left(\eta^{2}+|\nabla \eta|^{2}\right) v^{2} v_{L}^{2(s-1)} d x \\
& +C s \epsilon^{-\frac{2 N}{2 q-N}} \int_{\Omega}|x|^{-2(\sqrt{\bar{\mu}}-\sqrt{\bar{\mu}-\gamma})}\left|\eta v v_{L}^{s-1}\right|^{2} d x .
\end{aligned}
$$

Selecting $\epsilon=\frac{1}{\sqrt{2 C s}}$ we obtain

$$
\begin{aligned}
& \left(\int_{\Omega}|x|^{-2^{*}(\sqrt{\bar{\mu}}-\sqrt{\bar{\mu}-\gamma})}\left|\eta v v_{L}^{s-1}\right|^{2^{*}} d x\right)^{\frac{2}{2^{*}}} \\
& \quad \leq C s^{\alpha} \int_{\Omega}|x|^{-2(\sqrt{\bar{\mu}}-\sqrt{\bar{\mu}-\gamma})}\left(\eta^{2}+|\nabla \eta|^{2}\right) v^{2} v_{L}^{2(s-1)} d x
\end{aligned}
$$

where $\alpha=\frac{2 q}{2 q-N}$. We now observe that

$$
\int_{\Omega}|x|^{-2^{*}(\sqrt{\bar{\mu}}-\sqrt{\bar{\mu}-\gamma})}|\eta|^{2^{*}} v^{2} v_{L}^{2^{*} s-2} d x \leq \int_{\Omega}|x|^{-2^{*}(\sqrt{\bar{\mu}}-\sqrt{\bar{\mu}-\gamma})} \eta^{2^{*}} v^{2^{*}} v_{L}^{(s-1) 2^{*}} d x .
$$

Hence we derive from (34) that

$$
\begin{aligned}
\left(\int_{\Omega}|x|^{-2^{*}(\sqrt{\bar{\mu}}-\sqrt{\bar{\mu}-\gamma})} \eta^{2^{*}} v^{2} v_{L}^{2^{*} s-2} d x\right)^{\frac{2}{2^{*}}} & \\
& \leq C s^{\alpha} \int_{\Omega}|x|^{-2(\sqrt{\bar{\mu}}-\sqrt{\bar{\mu}-\gamma})}\left(\eta^{2}+|\nabla \eta|^{2}\right) v^{2} v_{L}^{2(s-1)} d x \\
& \leq C s^{\alpha} \int_{\Omega}|x|^{-2^{*}(\sqrt{\bar{\mu}}-\sqrt{\bar{\mu}-\gamma})}\left(\eta^{2}+|\nabla \eta|^{2}\right) v^{2} v_{L}^{2(s-1)} d x .
\end{aligned}
$$

From this, we deduce by the choice of the function $\eta$ that

$$
\begin{aligned}
\left(\int_{B(0, r) \cap \Omega}|x|^{-2^{*}(\sqrt{\bar{\mu}}-\sqrt{\bar{\mu}-\gamma})} v^{2} v_{L}^{2^{*} s-2} d x\right)^{\frac{2}{2^{*}}} & \\
& \leq \frac{C s^{\alpha}}{(\rho-r)^{2}} \int_{B(0, \rho) \cap \Omega}|x|^{-2^{*}(\sqrt{\mu}-\sqrt{\bar{\mu}-\gamma})} v^{2} v_{L}^{2(s-1)} d x .
\end{aligned}
$$

We now select $s^{*}$ such that $\frac{N}{N-2}<s^{*}$ with $\gamma\left(2 s^{*}+2\right)\left(\frac{1}{\bar{\mu}}+1\right) \leq 4 d_{1} S$ (see (27) in Proposition 3.2) which can be achieved by taking $\gamma_{0}$ smaller if necessary. Put 
$s_{n}=s^{*}\left(\frac{2^{*}}{2}\right)^{n}, n=0,1,2, \ldots$ We then obtain

$$
\begin{gathered}
\left(\int_{B(0, r) \cap \Omega}|x|^{-2^{*}(\sqrt{\bar{\mu}}-\sqrt{\bar{\mu}-\gamma})} v^{2} v_{L}^{2 s_{n+1}-2} d x\right)^{\frac{1}{2 s_{n}+1}} \\
\quad \leq\left(\frac{C s_{n}^{\alpha}}{(\rho-r)^{2}}\right)^{\frac{1}{2 s_{n}}}\left(\int_{B(0, \rho) \cap \Omega}|x|^{-2^{*}(\sqrt{\bar{\mu}}-\sqrt{\bar{\mu}-\gamma})} v^{2} v_{L}^{2\left(s_{n}-2\right)} d x\right)^{\frac{1}{2 s_{n}}}
\end{gathered}
$$

Let $\rho_{\circ}>0$ be small enough so that $a \notin B\left(0, \rho_{\circ}\right)$ and put $r_{n}=\rho_{\circ}\left(1+\rho_{\circ}^{n}\right), n=0,1,2, \ldots$ Inequality (35) with $\rho=r_{n}, r=r_{n+1}$ becomes

$$
\begin{gathered}
\left(\int_{B\left(0, r_{n+1}\right) \cap \Omega}|x|^{-2^{*}(\sqrt{\mu}-\sqrt{\bar{\mu}-\gamma})} v^{2} v_{L}^{2 s_{n+1}-2} d x\right)^{\frac{1}{2 s_{n+1}}} \\
\leq\left(\frac{C s_{n}^{\alpha}}{\left(\rho_{\circ}-\rho_{\circ}^{2}\right) \rho_{\circ}^{n}}\right)^{\frac{1}{2 s_{n}}}\left(\int_{B\left(0, r_{n}\right) \cap \Omega}|x|^{-2^{*}(\sqrt{\bar{\mu}}-\sqrt{\bar{\mu}-\gamma})} v^{2} v_{L}^{2 s_{n}-2} d x\right)^{\frac{1}{2 s_{n}}} .
\end{gathered}
$$

We now notice that

$$
\begin{aligned}
\int_{B\left(0, r_{\circ}\right)}|x|^{-2^{*}(\sqrt{\bar{\mu}}-\sqrt{\bar{\mu}-\gamma})} v^{2} v_{L}^{2 s^{*}-2} d x & \leq \int_{B\left(0, r_{\circ}\right) \cap \Omega}|x|^{\left(2 s^{*}-2^{*}\right)(\sqrt{\bar{\mu}}-\sqrt{\bar{\mu}-\gamma})}|u|^{2^{*}} d x \\
& \leq d^{\left(2 s^{*}-2^{*}\right)(\sqrt{\bar{\mu}}-\sqrt{\bar{\mu}-\gamma})} \int_{\Omega}|u|^{2 s^{*}} d x \leq C .
\end{aligned}
$$

Thus iterating (36) we see that

$$
\left\|v_{L}\right\|_{L^{2 s_{n+1}\left(B\left(0, \rho_{\circ}\right) \cap \Omega\right)}} \leq C
$$

for all $n$. For the computational details we refer to the paper $[6$, pp. 15, 16]. Letting $n \rightarrow \infty$ in (37) we obtain

$$
u(x) \leq C|x|^{-(\sqrt{\bar{\mu}}-\sqrt{\bar{\mu}-\gamma})} \quad \text { for } x \in\left(B\left(0, \rho_{\circ}\right)-\{0\}\right) \cap \Omega .
$$

To complete the proof, it remains to show that $u$ is bounded in $\Omega \cap B(a, \rho)$ for small $\rho>0$. We choose $\rho$ so that $0 \notin B(a, \rho)$. Again we use the Moser iteration technique. Let $\eta \in C_{\circ}^{1}(B(a, \rho))$ and satisfy $\eta=1$ on $B(a, r), r<\rho$, and $|\nabla \eta| \leq \frac{4}{\rho-r}$. We take as a test function $\phi=\eta^{2} u u_{L}^{2(s-1)}, L>0, s>1$. Testing (1) with $\phi$ we obtain

$$
\begin{aligned}
\int_{\Omega}\left(2 \eta u u_{L}^{2(s-1)} \nabla \eta \nabla u\right. & \left.+\eta^{2} u_{L}^{2(s-1)}|\nabla u|^{2}+2(s-1) \eta^{2} u_{L}^{2(s-1)}\left|\nabla u_{L}\right|^{2}\right) d x \\
& +\frac{\lambda}{d^{2}} \int_{\Omega} u^{2} \eta^{2} u_{L}^{2(s-1)} d x \\
\leq & Q_{M} \int_{\Omega} \eta^{2}|u|^{2^{*}} u_{L}^{2(s-1)} d x+\gamma C \int_{\Omega} u^{2} \eta^{2} u_{L}^{2(s-1)} d x
\end{aligned}
$$


For every $\epsilon>0$ we have

$$
2 \int_{\Omega} \eta u u_{L}^{2(s-1)} \nabla \eta \nabla u d x \leq \epsilon \int_{\Omega} \eta^{2}\left|u_{L}\right|^{2(s-1)}|\nabla u|^{2} d x+C(\epsilon) \int_{\Omega}|\nabla \eta|^{2} u^{2} u_{L}^{2(s-1)} d x .
$$

Taking $\epsilon=\frac{1}{2}$ we derive from (38) that

$$
\begin{aligned}
& \int_{\Omega}\left(\eta^{2} u_{L}^{2(s-1)}|\nabla u|^{2}\right.\left.+4(s-1) \eta^{2} u_{L}^{2(s-1)}\left|\nabla u_{L}\right|^{2}\right) d x+\frac{\lambda}{d^{2}} \int_{\Omega} u^{2} \eta^{2} u_{L}^{2(s-1)} d x \\
& \leq C\left(\int_{\Omega}\left(\eta^{2}+|\nabla \eta|^{2}\right) u^{2} u_{L}^{2(s-1)} d x+\int_{\Omega}|u|^{2^{*}} \eta^{2} u_{L}^{2(s-1)} d x\right) .
\end{aligned}
$$

According to Proposition 3.2 we can choose $q$ with $\left(2^{*}-2\right) q>1$ and $2<\frac{2 q}{q-1}<2^{*}$ such that $u \in L^{q\left(2^{*}-2\right)}(\Omega)$. We then have

$$
\begin{aligned}
\int_{\Omega} \eta^{2}|u|^{2^{*}} u_{L}^{2(s-1)} d x \leq & \|u\|_{L^{\left(2^{*}-2\right) q}(\Omega)}^{2^{*}-2}\left\|\eta u u_{L}^{s-1}\right\|_{L^{\frac{2 q}{q-1}}(\Omega)}^{2} \\
\leq & \|u\|_{L^{\left(2^{*}-2\right) q}(\Omega)}^{2^{*}-2}\left(\epsilon\left\|\eta u u_{L}^{s-1}\right\|_{L^{2^{*}}(\Omega)}\right. \\
& \left.+C(N, q) \epsilon^{-\frac{N}{2 q-N}}\left\|\eta u u_{L}^{(s-1)}\right\|_{L^{2}(\Omega)}\right)^{2} \\
\leq & C \epsilon^{2}\left(\int_{\Omega}\left|\eta u u_{L}^{s-1}\right|^{2^{*}} d x\right)^{\frac{2}{2^{*}}}+C \epsilon^{-\frac{2 N}{2 q-N}} \int_{\Omega}\left|\eta u u_{L}^{s-1}\right|^{2} d x .
\end{aligned}
$$

Inserting this into (39) and using the Sobolev inequality we obtain

$$
\begin{aligned}
\left(\int_{\Omega}\left|\eta u u_{L}^{s-1}\right|^{2^{*}} d x\right)^{\frac{2}{2^{*}}} \leq & C s \epsilon^{2}\left(\int_{\Omega}\left|\eta u u_{L}^{s-1}\right|^{2^{*}} d x\right)^{\frac{2}{2^{*}}} \\
& +C s \int_{\Omega}\left(\eta^{2}+|\nabla \eta|^{2}\right) u^{2} u_{L}^{2(s-1)} d x \\
& +C s \epsilon^{-\frac{2 N}{2 q-N}} \int_{\Omega}\left|\eta u u_{L}^{s-1}\right|^{2} d x .
\end{aligned}
$$

The proof is completed using the Moser iteration technique.

\section{Existence of sign-changing solutions}

For $\delta>0$ small we we consider the following perturbed problem

$$
\begin{cases}-\Delta u+\lambda \frac{u}{|x-a|^{2}}-\gamma \frac{u}{|x|^{2}} & =Q(x)|u|^{2^{*}-2-\delta} \text { in } \Omega \\ \frac{\partial u}{\partial \nu} & =0 \text { on } \partial \Omega, \quad u>0 \text { on } \Omega .\end{cases}
$$

The variational functional for problem (40) is given by

$$
J_{\lambda, \gamma, \delta}(u)=\frac{1}{2} \int_{\Omega}\left(|\nabla u|^{2}+\frac{\lambda u^{2}}{|x-a|^{2}}-\frac{\gamma u^{2}}{|x|^{2}}\right) d x-\frac{1}{2^{*}-\delta} \int_{\Omega} Q(x)|u|^{2^{*}-\delta} d x
$$


for $u \in H^{1}(\Omega)$. Since problem (40) is subcritical, the functional $J_{\lambda, \gamma, \delta}$ satisfies the (PS) condition. In what follows, we will use some ideas from paper [29], where problem (40) with $\lambda=\gamma=0$ and $Q$ constant was studied. Let $\Lambda_{\delta}$ be a Nehari manifold for $J_{\lambda, \gamma, \delta}$

$$
\Lambda_{\delta}=\left\{u \neq 0, u \in H^{1}(\Omega) ;\left\langle J_{\lambda, \gamma, \delta}^{\prime}(u), u\right\rangle=0\right\} .
$$

We set

$$
c_{1, \delta}=\inf _{u \in \Lambda_{\delta}} J_{\lambda, \gamma, \delta}(u) .
$$

If $\delta=0$ then $\Lambda_{0}$ becomes the Nehari manifold for the functional $J_{\lambda, \gamma}$ and we set $c_{1,0}=\inf _{u \in \Lambda_{0}} J_{\lambda, \gamma}(u)$. It is known that

$$
c_{1,0}=J_{\lambda, \gamma}\left(u_{1}\right)=\inf _{u \in \Lambda_{0}} J_{\lambda, \gamma}(u),
$$

where $u_{1}$ is a solution of (1) constructed in section 3. As in paper [29], we can show that

$$
\lim _{\delta \rightarrow 0} c_{1, \delta}=c_{1,0} .
$$

Since the variational problem of finding a minimizer of $J_{\lambda, \gamma, \delta}$ on $\Lambda_{\delta}$ is subcritical it is easy to show that there exists a minimizer of $J_{\lambda, \gamma, \delta}$ on $\Lambda_{\delta}$ for $\gamma \leq \gamma_{\circ}$, where $\gamma_{\circ}$ is a constant from Proposition 1.5. To establish the existence of sign-changing solutions of (1), we use the Lusternik-Schnirelmann [3] theory of critical points of even functionals.

We recall the definition of Krasnoselski genus. Let $A \subset H^{1}(\Omega)$ be a closed, bounded set which is $\mathbb{Z}_{2}$-symmetric (i.e., $u \in A \Rightarrow-u \in A$ ). The Krasnoselski genus is defined by

$$
i(A)=\inf \left\{n, \quad \text { there exists an odd and continuous map } h: A \rightarrow \mathbb{R}^{n}-\{0\}\right\} .
$$

For a fixed $\rho>0$ we put $S_{\rho}=\left\{u \in H^{1}(\Omega) ;\|u\|=\rho\right\}$ and define

$$
\mathcal{H}=\left\{h: H^{1}(\Omega) \rightarrow H^{1}(\Omega) ; h \text { is an odd homeomorphism }\right\}
$$

and

$$
\mathcal{F}_{2}=\left\{A \text { closed, } \mathbb{Z}_{2} \text {-symmetric; } i\left(h(A) \cap S_{\rho}\right) \geq 2 \text { for every } h \in \mathcal{H}\right\} .
$$

Let

$$
I_{\lambda, \gamma}(u)=\int_{\Omega}\left(|\nabla u|^{2}+\frac{\lambda u^{2}}{|x-a|^{2}}-\frac{\gamma u^{2}}{|x|^{2}}\right) d x .
$$

Proposition 4.1. Let $\gamma \leq \gamma_{0}$. For every $p \in\left(2,2^{*}\right)$ and $u \in L^{p}(\Omega)-\{0\}$ there exists $v=v(u) \in H^{1}(\Omega)$ such that

$$
\int_{\Omega} Q(x)|u|^{p-2} v^{2} d x=1, \quad v(x) \geq 0 \quad \text { on } \Omega
$$

and

$$
\mu_{1}(u):=I_{\lambda, \gamma}(v)=\inf \left\{I_{\lambda, \gamma}(w) ; w \in H^{1}(\Omega), \quad \int_{\Omega} Q(x)|u|^{p-2} w^{2} d x=1\right\} .
$$


Since $H^{1}(\Omega)$ is compactly embedded into $L^{p}(\Omega), 2 \leq p<2^{*}$, it is easy to show that a minimizer for $I_{\lambda, \gamma}$ exists. Obviously $\mu_{1}(u)$ is the first eigenvalue with the eigenfunction $v(u)$ of the problem

$$
\begin{cases}-\Delta v+\frac{\lambda}{|x-a|^{2}} v-\frac{\gamma}{|x|^{2}} v & =\mu_{1}(u) Q(x)|u|^{p-2} v \text { in } \Omega, \\ \frac{\partial v}{\partial \nu} & =0 \text { on } \partial \Omega .\end{cases}
$$

Proposition 4.2. Let $\gamma \leq \gamma_{0}$. For every $\delta \in\left(0, \frac{4}{N-2}\right)$ there exists a nontrivial solution $u_{\delta}$ of the Neumann problem

$$
\begin{cases}-\Delta u+\frac{\lambda u}{|x-a|^{2}}-\frac{\gamma u}{|x|^{2}} & =Q(x)|u|^{2^{*}-\delta-2} u \text { in } \Omega, \\ \frac{\partial u}{\partial \nu}= & 0 \text { on } \partial \Omega,\end{cases}
$$

which satisfies

$$
\int_{\Omega}\left|u_{\delta}\right|^{2^{*}-\delta-2} u_{\delta} v\left(u_{\delta}\right) d x=0,
$$

where $v\left(u_{\delta}\right)$ is defined in Proposition 4.1 with $p=2^{*}-\delta$.

The main idea of the proof is to construct the (PS) ${ }_{c}$ sequence for the lever $c_{2, \delta}=$ $\inf _{A \in \mathcal{F}_{2}} \sup _{u \in A} J_{\lambda, \gamma, \delta}(u)$. The proof of this fact is similar to that of in [14, Theorem 1'; 29, Proposition 1.2]. Since the problem is subcritical, the Palais-Smale condition holds and we also have

$$
J_{\lambda, \gamma, \delta}\left(u_{\delta}\right)=c_{2, \delta}=\inf _{A \in \mathcal{F}_{2}} \sup _{u \in A} J_{\lambda, \gamma, \delta}(u) .
$$

We need the following estimates for $U_{\epsilon, y}$ with $y \neq 0, a$

$$
\begin{aligned}
& \int_{\Omega} u_{1} U_{\epsilon, y}^{2^{*}-\delta-1} d x=O\left(\epsilon^{\frac{N-2}{2}}\right), \\
& \int_{\Omega} u_{1}^{2^{*}-\delta-1} U_{\epsilon, y} d x=O\left(\epsilon^{\frac{N-2}{2}}\right)
\end{aligned}
$$

and

$$
\int_{\Omega} u_{1}^{2^{*}-1} U_{\epsilon, y} d x=O\left(\epsilon^{\frac{N-2}{2}}\right) .
$$

We only give the proof of (44). Let $\rho>0$. It follows from Theorem 3.4 that

$$
\begin{aligned}
\int_{\Omega} u_{1}^{2^{*}-\delta-1} U_{\epsilon, y} d x \leq & C \int_{\Omega}|x|^{-(\sqrt{\bar{\mu}}-\sqrt{\bar{\mu}-\gamma})\left(2^{*}-1-\delta\right)} U_{\epsilon, y} d x \\
\leq & C \int_{B(y, \rho) \cap \Omega}|x|^{-(\sqrt{\bar{\mu}}-\sqrt{\bar{\mu}-\gamma})\left(2^{*}-\delta-1\right)} U_{\epsilon, y} d x \\
& +C \int_{\mathbb{R}^{N}-B(y, \rho) \cap \Omega}|x|^{-(\sqrt{\bar{\mu}}-\sqrt{\mu}-\gamma)\left(2^{*}-1-\delta\right)} U_{\epsilon, y} d x=J_{1}+J_{2} .
\end{aligned}
$$


We may assume that $|y|>\rho$. Then

$$
\begin{aligned}
\left|J_{1}\right| & \leq \int_{|z|<\frac{\rho}{\epsilon}} \frac{\epsilon^{\frac{N+2}{2}}}{|z \epsilon+y|^{(\sqrt{\mu}-\sqrt{\bar{\mu}-\gamma})\left(2^{*}-\delta-1\right)}\left(1+|z|^{2}\right)^{\frac{N-2}{2}}} d x \\
& \leq \int_{|z|<\frac{\rho}{\epsilon}} \frac{\epsilon^{\frac{N+2}{2}}}{(|y|-\rho)^{(\sqrt{\mu}-\sqrt{\bar{\mu}-\gamma})\left(2^{*}-1-\delta\right)}\left(1+|z|^{2}\right)^{\frac{N-2}{2}}} d z=O\left(\epsilon^{\frac{N-2}{2}}\right) .
\end{aligned}
$$

For the integral $J_{2}$ we have the estimate

$$
\left|J_{2}\right| \leq C \epsilon^{\frac{N-2}{2}} \int_{\Omega}|x|^{-(\sqrt{\bar{\mu}}-\sqrt{\bar{\mu}-\gamma})\left(2^{*}-1-\delta\right)} d x .
$$

This integral is convergent for $0<\gamma \leq \gamma_{0}$. (We may take $\gamma_{0}$ smaller if necessary.) In a similar way we establish estimates (43) and (45).

Lemma 4.3. Let $N \geq 5,0<\gamma \leq \gamma_{\circ}$ and $c^{*}=\frac{S^{\frac{N}{2}}}{2 N Q_{m^{\frac{N-2}{2}}}^{\frac{N-}{2}}}$ Suppose that $Q_{m}=Q(y)$, $y \neq 0, a$, and

$$
|Q(x)-Q(y)|=o(|x-y|) \quad \text { for } x \text { near } y
$$

with $H(y)>0$. Then there exists $\sigma>0$ and $\delta_{\circ}>0$ such that

$$
c_{2, \delta} \leq c_{1, \delta}+c^{*}-\sigma
$$

for $\delta \leq \delta_{\circ}$

Proof. Let $A=\operatorname{span}\left\{u_{1}, U_{\epsilon, y}\right\}$. Since $A \in \mathcal{F}_{2}$ we have

$$
c_{2, \delta} \leq \sup _{u \in A} J_{\lambda, \gamma, \delta}(u)=\sup _{s, t \in \mathbb{R}} J_{\lambda, \gamma, \delta}\left(s u_{1}+t U_{\epsilon, y}\right) .
$$

Since $\lim _{|s|,|t| \rightarrow \infty} J_{\lambda, \gamma, \delta}\left(s u_{1}+t U_{\epsilon, y}\right)=-\infty$, we can assume that $|s|+|t| \leq C$ for some constant $C>0$. To proceed further we use the following inequality: for $a, b \in \mathbb{R}$ and $q \geq 1$ we have

$$
|a+b|^{q} \geq|a|^{q}+|b|^{q}-C\left(|a|^{q-1}|b|+|a||b|^{q-1}\right),
$$


where $C>0$ is independent of $a$ and $b$. We then have

$$
\begin{aligned}
& J_{\lambda, \gamma, \delta}\left(s u_{1}+t U_{\epsilon, y}\right) \\
&=\frac{s^{2}}{2}\left\|\nabla u_{1}\right\|_{2}^{2}+\frac{t^{2}}{2}\left\|\nabla U_{\epsilon, y}\right\|_{2}^{2}+s t \int_{\Omega} \nabla u_{1} \nabla U_{\epsilon, y} d x \\
&+\frac{\lambda s^{2}}{2} \int_{\Omega} \frac{u_{1}^{2}}{|x-a|^{2}} d x+\frac{\lambda t^{2}}{2} \int_{\Omega} \frac{U_{\epsilon, y}^{2}}{|x-a|^{2}} d x+\lambda s t \int_{\Omega} \frac{u_{1} U_{\epsilon, y}}{|x-a|^{2}} d x \\
& \quad-\frac{\gamma s^{2}}{2} \int_{\Omega} \frac{u_{1}^{2}}{|x|^{2}} d x-\frac{\gamma t^{2}}{2} \int_{\Omega} \frac{U_{\epsilon, y}^{2}}{|x|^{2}} d x-s t \gamma \int_{\Omega} \frac{u_{1} U_{\epsilon, y}}{|x|^{2}} d x \\
&-\frac{1}{2^{*}-\delta} \int_{\Omega} Q(x)\left|s u_{1}+t U_{\epsilon, y}\right|^{2^{*}-\delta} d x \\
&= s t \int_{\Omega} Q(x) u_{1}^{2^{*}-1} U_{\epsilon, y} d x+J_{\lambda, \gamma}\left(s u_{1}\right)+\frac{s^{2^{*}}}{2^{*}} \int_{\Omega} Q(x)\left|u_{1}\right|^{2^{*}} d x \\
&+J_{\lambda, \gamma}\left(t U_{\epsilon, y}\right)+\frac{|t|^{2^{*}}}{2^{*}} \int_{\Omega} Q(x) U_{\epsilon, y}^{2^{*}} d x \\
&-\frac{1}{2^{*}-\delta} \int_{\Omega} Q(x)\left|s u_{1}+t U_{\epsilon, y}\right|^{2^{*}-\delta} d x \\
& \leq|s t| \int_{\Omega} Q(x) u_{1}^{2^{*}-1} U_{\epsilon, y} d x+c_{1,0}+J_{\lambda, \gamma}\left(t U_{\epsilon, y}\right) \\
&+\frac{|s|^{2^{*}}}{2^{*}} \int_{\Omega} Q(x) u_{1}^{2^{*}} d x-\frac{|s|^{2^{*}-\delta}}{2^{*}-\delta} \int_{\Omega} Q(x) u_{1}^{2^{*}-\delta} d x+\frac{|t|^{2^{*}}}{2^{*}} \int_{\Omega} Q(x) U_{\epsilon, y}^{2^{*}} d x \\
&-\frac{|t|^{2^{*}-\delta}}{2^{*}-\delta} \int_{\Omega} Q(x) U_{\epsilon, y}^{2^{*}-\delta} d x+C \int_{\Omega} u_{1}^{2^{*}-1-\delta} U_{\epsilon, y} d x+C \int_{\Omega} u_{1} U_{\epsilon, y}^{2^{*}-1-\delta} d x .
\end{aligned}
$$

By (43), (44), (45), (46), and (20) we get

$$
\begin{aligned}
J_{\lambda, \gamma, \delta}\left(s u_{1}+t U_{\epsilon, y}\right) \leq & \frac{S^{\frac{N}{2}}}{2 N Q_{m^{*}}^{\frac{N-2}{2}}}-c_{1} \epsilon+c_{1,0} \\
& +\frac{|s|^{2^{*}}}{2^{*}} \int_{\Omega} Q(x)\left|u_{1}\right|^{2^{*}} d x-\frac{|s|^{2^{*}-\delta}}{2^{*}-\delta} \int_{\Omega} Q(x)\left|u_{1}\right|^{2^{*}-\delta} d x \\
& +\frac{|t|^{2^{*}}}{2^{*}} \int_{\Omega} Q(x) U_{\epsilon, y}^{2^{*}} d x-\frac{|t|^{2^{*}-\delta}}{2^{*}-\delta} \int_{\Omega} Q(x) U_{\epsilon, y}^{2^{*}-\delta} d x
\end{aligned}
$$

for $\epsilon \leq \epsilon_{\circ}$ and some constant $c_{1}>0$. Since $c_{1, \delta} \rightarrow c_{1,0}$ and the last two differences tend to 0 for every $0<\epsilon \leq \epsilon_{\circ}$ the result follows.

Theorem 4.4. Suppose that the assumptions of Lemma 4.3 hold. Then problem (1) has a sign-changing solution. 
Proof. Let $\left\{u_{\delta}\right\}, \delta \leq \delta_{\circ}$ be solutions of (41). It is clear that $\left\|u_{\delta}\right\|_{H^{1}(\Omega)} \leq C$ for $\delta \in\left(0, \delta_{\circ}\right)$ and some constant $C>0$ independent of $\delta$. Let $u_{\delta}^{+}(x)=\max \left(0, u_{\delta}(x)\right)$ and $u_{\delta}^{-}(x)=\max \left(0,-u_{\delta}(x)\right)$. By $(42) u_{\delta}^{+} \neq 0$ and $u_{\delta}^{-} \neq 0$. We also have $\left\|u_{\delta}^{+}\right\|_{H^{1}}$, $\left\|u_{\delta}^{-}\right\|_{H^{1}} \leq C$. We may assume that up to a subsequence $u_{\delta}^{+} \rightarrow u^{+}$and $u_{\delta} \rightarrow u^{-}$ in $H^{1}(\Omega)$. Since $u_{\delta}^{+}, u_{\delta}^{-} \in \Lambda_{\delta}$ we have $J_{\lambda, \gamma, \delta}\left(u_{\delta}^{+}\right), J_{\lambda, \gamma, \delta}\left(u_{\delta}^{-}\right) \geq c_{1, \delta}$. Since $c_{1, \delta} \rightarrow c_{1,0}$ as $\delta \rightarrow 0$, by Lemma 4.3 , we have

$$
J_{\lambda, \gamma, \delta}\left(u_{\delta}^{+}\right)+J_{\lambda, \gamma, \delta}\left(u_{\delta}^{-}\right)=J_{\lambda, \gamma, \delta}\left(u_{\delta}\right)=c_{2, \delta} \leq c_{1, \delta}+c^{*}-\sigma .
$$

Hence $J_{\lambda, \gamma, \delta}\left(u_{\delta}^{+}\right) \leq c^{*}-\sigma$ and moreover

$$
k_{1} \leq\left\|u_{\delta}^{+}\right\|_{L^{2 *}(\Omega)} \leq k_{2}
$$

for some constants $k_{1}>0$ and $k_{2}>0$ independent of $\delta \leq \delta_{\circ}$. We show that $u^{+} \neq 0$. Arguing by contradiction, assume $u^{+} \equiv 0$ on $\Omega$. By the concentration-compactness principle (see the proof of Proposition 1.4) we get

$$
\left|\nabla u_{\delta}^{+}\right|^{2} \rightarrow d \mu \geq \sum_{j \in \mathcal{J}} \mu_{j} \delta_{x_{j}}+\mu_{a} \delta_{a}+\mu_{0} \delta_{0}
$$

and

$$
\begin{gathered}
\left|u_{\delta}^{+}\right|^{2^{*}} \rightarrow d \nu=\sum_{j \in \mathcal{J}} \nu_{j} \delta_{x_{j}}+\nu_{a} \delta_{a}+\nu_{0} \delta_{0} \\
\frac{\left(u_{\delta}^{+}\right)^{2}}{|x-a|^{2}} \rightarrow d \tilde{\gamma}=\gamma_{a} \delta_{a}, \quad \text { and } \quad \frac{u_{\delta}^{+}}{|x|^{2}} \rightarrow d \tilde{\lambda}=\lambda_{0} \delta_{0}
\end{gathered}
$$

with $\mu_{j}, \nu_{j}, \mu_{a}, \nu_{a}, \mu_{0}$, and $\nu_{0}$ satisfying (9)-(12). As in the proof of Proposition 1.4 we show that $\nu_{x_{j}}=0, j \in \mathcal{J}, \nu_{a}=\nu_{0}=0$. This yields $\lim _{\delta \rightarrow 0} \int_{\Omega} Q(x)\left|u_{\delta}^{+}\right|^{2^{*}} d x=0$ which contradicts (47). In a similar manner we show that $u^{-} \neq 0$. Therefore $u$ is a sign-changing solution of (1). We show that $u_{\delta} \rightarrow u$ up to a subsequence in $H^{1}(\Omega)$. Put $w_{\delta}=u_{\delta}-u$. By Brézis-Lieb lemma [4] we have

$$
c_{1, \delta}+c^{*}-\sigma \geq J_{\lambda, \gamma, \delta}\left(u+w_{\delta}\right)=c_{1,0}+J_{\lambda, \gamma, \delta}\left(w_{\delta}\right)+o(1) .
$$

Since $\lim _{\delta \rightarrow 0} c_{1, \delta}=c_{1,0}$ we see that

$$
\begin{aligned}
\frac{1}{2} \int_{\Omega}\left(\left|\nabla w_{\delta}\right|^{2}+\frac{\lambda w_{\delta}^{2}}{|x-a|^{2}}-\frac{\gamma w_{\delta}^{2}}{|x|^{2}}\right) & d x \\
& -\frac{1}{2^{*}-\delta} \int_{\Omega} Q(x)\left|w_{\delta}\right|^{2^{*}-\delta} d x \leq c^{*}-\sigma+o(1) .
\end{aligned}
$$

Repeating the proof of the previous part, we derive that

$$
\lim _{\delta \rightarrow 0} \int_{\Omega} Q(x)\left|w_{\delta}\right|^{2^{*}} d x=0 .
$$


We now observe that

$$
0=\left\langle J_{\lambda, \gamma, \delta}^{\prime}\left(u_{\delta}\right), u_{\delta}\right\rangle=\left\langle J_{\lambda, \gamma, \delta}^{\prime}(u), u\right\rangle+\left\langle J_{\lambda, \gamma, \delta}^{\prime}\left(w_{\delta}\right), w_{\delta}\right\rangle=\left\langle J_{\lambda, \gamma, \delta}^{\prime}\left(w_{\delta}\right), w_{\delta}\right\rangle+o(1) .
$$

It then follows from Lemma 1.1, (48), and (49) that for every $\epsilon>0$ small, we have

$$
\begin{aligned}
\int_{\Omega}\left(\left|\nabla w_{\delta}\right|^{2}+\frac{\lambda w_{\delta}^{2}}{|x-a|^{2}}\right) d x & \leq \gamma\left(\frac{1}{\bar{\mu}}+\epsilon\right) \int_{\Omega}\left(\left|\nabla w_{\delta}\right|^{2}+C(\epsilon) w_{\delta}^{2}\right) d x \\
& =\gamma\left(\frac{1}{\bar{\mu}}+\epsilon\right) \int_{\Omega}\left|\nabla w_{\delta}\right|^{2} d x+C(\epsilon) o(1)
\end{aligned}
$$

Since $\gamma \leq \gamma_{0}$, we deduce from this that $w_{\delta} \rightarrow 0$ in $H^{1}(\Omega)$, that is, $u_{\delta} \rightarrow u$ in $H^{1}(\Omega)$.

\section{References}

[1] Adimurthi and G. Mancini, The Neumann problem for elliptic equations with critical nonlinearity, Nonlinear Analysis: A tribute in honour of G. Prodi, Sc. Norm. Super. di Pisa Quaderni, Scuola Norm. Sup., Pisa, 1991, pp. 9-25.

[2] - Geometry and topology of the boundary in the critical Neumann problem, J. Reine Angew. Math. 456 (1994), 1-18.

[3] A. Ambrosetti and P. H. Rabinowitz, Dual variational methods in critical point theory and applications, J. Functional Analysis 14 (1973), 349-381.

[4] H. Brézis and E. Lieb, A relation between pointwise convergence of functions and convergence of functionals, Proc. Amer. Math. Soc. 88 (1983), no. 3, 486-490.

[5] H. Brézis and T. Kato, Remarks on the Schrödinger operator with singular complex potentials, J. Math. Pures Appl. (9) 58 (1979), no. 2, 137-151.

[6] D. Cao and P. Han, Solutions to critical elliptic equations with multi-singular inverse square potentials, J. Differential Equations 224 (2006), no. 2, 332-372.

[7] Solutions for semilinear elliptic equations with critical exponents and Hardy potential, J. Differential Equations 205 (2004), no. 2, 521-537.

[8] L. Caffarelli, R. Kohn, and L. Nirenberg, First order interpolation inequalities with weights, Compositio Math. 53 (1984), no. 3, 259-275.

[9] F. Catrina and Z.-Q. Wang, On the Caffarelli-Kohn-Nirenberg inequalities: sharp constants, existence (and nonexistence), and symmetry of extremal functions, Comm. Pure Appl. Math. 54 (2001), no. 2, 229-258.

[10] J. Chabrowski, On the nonlinear Neumann problem involving the critical Sobolev exponent and Hardy potential, Rev. Mat. Complut. 17 (2004), no. 1, 195-227.

[11] J. Chabrowski and M. Willem, Least energy solutions of a critical Neumann problem with a weight, Calc. Var. Partial Differential Equations 15 (2002), no. 4, 421-431.

[12] J. Chen, Multiple positive solutions for a semilinear equation with prescribed singularity, J. Math. Anal. Appl. 305 (2005), no. 1, 140-157.

[13] K. S. Chou and C. W. Chu, On the best constant for a weighted Sobolev-Hardy inequality, J. London Math. Soc. (2) 48 (1993), no. 1, 137-151. 
[14] M. Comte and G. Tarantello, A Neumann problem with critical Sobolev exponent, Houston J. Math. 18 (1992), no. 2, 279-294.

[15] D. G. Costa and O. H. Miyagaki, On a class of critical elliptic equations of Caffarelli-KohnNirenberg type, Contributions to nonlinear analysis, Progr. Nonlinear Differential Equations Appl., vol. 66, Birkhäuser, Basel, 2006, pp. 207-220.

[16] I. Ekeland and N. Ghoussoub, Selected new aspects of the calculus of variations in the large, Bull. Amer. Math. Soc. (N.S.) 39 (2002), no. 2, 207-265.

[17] A. Ferrero and F. Gazzola, Existence of solutions for singular critical growth semilinear elliptic equations, J. Differential Equations 177 (2001), no. 2, 494-522.

[18] V. Felli and S. Terracini, Elliptic equations with multi-singular inverse-square potentials and critical nonlinearity, Comm. Partial Differential Equations 31 (2006), no. 1-3, 469-495.

[19] N. Ghoussoub and X. S. Kang, Hardy-Sobolev critical elliptic equations with boundary singularities, Ann. Inst. H. Poincaré Anal. Non Linéaire 21 (2004), no. 6, 767-793.

[20] N. Ghoussoub and C. Yuan, Multiple solutions for quasi-linear PDEs involving the critical Sobolev and Hardy exponents, Trans. Amer. Math. Soc. 352 (2000), no. 12, 5703-5743.

[21] D. Gilbarg and N. S. Trudinger, Elliptic partial differential equations of second order, 2nd ed., Grundlehren der Mathematischen Wissenschaften, vol. 224, Springer-Verlag, Berlin, 1983.

[22] P. Han, Quasilinear elliptic problems with critical exponents and Hardy terms, Nonlinear Anal. 61 (2005), no. 5, 735-758.

[23] P. Han and Z. Liu, Positive solutions for elliptic equations involving critical Sobolev exponents and Hardy terms with Neumann boundary conditions, Nonlinear Anal. 55 (2003), no. 1-2, 167186.

[24] E. Jannelli, The role played by space dimension in elliptic critical problems, J. Differential Equations 156 (1999), no. 2, 407-426.

[25] D. Kang and S. Peng, Existence of solutions for elliptic problems with critical Sobolev-Hardy exponents, Israel J. Math. 143 (2004), 281-297.

[26] P.-L. Lions, The concentration-compactness principle in the calculus of variations: The limit case. I, Rev. Mat. Iberoamericana 1 (1985), no. 1, 145-201; II, Rev. Mat. Iberoamericana 1 (1985), no. 2, 45-120.

[27] W.-M. Ni and I. Takagi, On the shape of least-energy solutions to a semilinear Neumann problem, Comm. Pure Appl. Math. 44 (1991), no. 7, 819-851.

[28] D. Smets, Nonlinear Schrödinger equations with Hardy potential and critical nonlinearities, Trans. Amer. Math. Soc. 357 (2005), no. 7, 2909-2938.

[29] G. Tarantello, Nodal solutions of semilinear elliptic equations with critical exponent, Differential Integral Equations 5 (1992), no. 1, 25-42.

[30] S. Terracini, On positive entire solutions to a class of equations with a singular coefficient and critical exponent, Adv. Differential Equations 1 (1996), no. 2, 241-264.

[31] X. J. Wang, Neumann problems of semilinear elliptic equations involving critical Sobolev exponents, J. Differential Equations 93 (1991), no. 2, 283-310. 